\title{
Estimation de la concentration en hydrocarbures totaux du sol d'un ancien site pétrochimique : étude méthodologique
}

\author{
C. de Fouquet ${ }^{1}$, A. Prechtel ${ }^{1,2}$ et J.C. Setier ${ }^{3}$ \\ 1 Centre de Géostatistique, École des mines de Paris, 35, rue Saint-Honoré, 77305 Fontainebleau Cedex - France \\ 2 Actuellement : Institut für Angewandte Mathematik, Universität Erlangen-Nürnberg, Martensstr. 3, 91058 Erlangen - Allemagne \\ 3 Total Exploration-Production, Département Environnement-Projets, 64018 Pau Cedex - France \\ e-mail : chantal.de_fouquet@ensmp.fr - alexander.prechtel@am.uni-erlangen.de-jean-claude.setier@total.com
}

\begin{abstract}
Résumé — L'analyse variographique exploratoire des mesures en hydrocarbures totaux dans les sols d'un ancien site pétrochimique met en évidence l'hétérogénéité de la pollution. La bimodalité de l'histogramme indique deux classes de concentration, différentes tant par leur niveau que par leur variabilité spatiale. Dans cet article, nous montrons comment un examen détaillé des données apporte une meilleure compréhension de la pollution sur le site, révélant par exemple un remaniement local des sols. La validation croisée, utilisée pour comparer quantitativement trois modèles géostatistiques de complexité croissante, montre la nécessité d'une modélisation détaillée de la variabilité spatiale des concentrations. Elle fournit également des indications sur les incertitudes, notamment pour l'estimation des fortes concentrations. Supposant ensuite une sélection «ponctuelle», nous calculons le « récupérable (quantité de polluant, concentration moyenne) prévu et effectif pour chacun des trois modèles, et nous quantifions l'effet d'information résultant des inévitables erreurs de sélection. Une méthode simple et rigoureuse de calcul de la probabilité de dépassement de seuil ou des quantiles locaux de l'histogramme des concentrations est présentée.
\end{abstract}

\footnotetext{
Abstract - Estimation of the Total Hydrocarbon Concentration in the Soil of a Former Petrochemical Site: Methodological Study - The exploratory variographic analysis of the total hydrocarbon concentration measurements in the soil of a former petrochemical implant, highlights the heterogeneity of the pollution. The bimodality on the histogram indicates the presence of two classes of concentration, which represent different spatial structures. We show how a detailed examination of the data can reveal, for example, a local backfill. Cross validation, allowing to compare measured and estimated concentration values at sampling points, is used to show that taking into account this spatial heterogeneity is necessary to improve the estimation. It also makes it possible to evaluate uncertainties, in particular on the estimation of high concentrations. Considering the case of a punctual selection, we compare at different threshold, estimated and effective recovering reserves (quantity of pollutant, mean concentration upon threshold). The consequences of selection errors due to the selection on estimators instead on true values are quantified, in relation with the model of the spatial structure. A rigorous and efficient method to calculate the probability that the concentration exceeds a given threshold, and the quantiles of the local distribution, is presented.
} 


\section{INTRODUCTION}

Que ce soit pour évaluer correctement le terme source dans les études de risques (étude du transfert vers la nappe ou quantification de niveaux d'exposition notamment), pour déterminer les procédés de traitement adaptés (atténuation naturelle contrôlée ou traitement physicochimique après excavation, par exemple), ou pour contrôler les opérations de dépollution, une connaissance précise du niveau de concentration des polluants présents dans les sols d'anciens sites industriels est indispensable. C'est pourquoi, depuis une quinzaine d'années, l'estimation géostatistique a été peu à peu utilisée pour quantifier les volumes et les concentrations des sols pollués, d'abord à l'échelle régionale (Leenaers et al., 1989; Schloz et al., 1999), puis à l'échelle du site. Dans ce contexte, différentes méthodes géostatistiques d'estimation linéaire ou non linéaire ont été validées par des études méthodologiques (Papritz et Dubois, 1999).

Dans le cas d'un traitement sélectif des sols, qui nécessite de délimiter localement les zones à dépolluer suivant leur concentration, il est indispensable de vérifier que les procédures d'échantillonnage et d'estimation prévues pour guider la dépollution sont effectivement compatibles avec la variabilité spatiale des concentrations. En effet, un fort contraste des concentrations à l'échelle métrique à décamétrique peut avoir comme conséquence une estimation très imprécise de la concentration de blocs de quelques mètres cubes. Cette imprécision rend alors illusoires les procédures de sélection, même en présence d'un échantillonnage très dense du chantier.

Or, les concentrations en polluants organiques mesurées dans les sols d'anciens sites industriels recouvrent généralement une gamme étendue de valeurs, et présentent localement de forts contrastes entre prélèvements voisins. La structure spatiale apparaît alors médiocre, et les estimations sont peu précises (Jeannée, 2001).

Dans ce contexte, l'échantillonnage localement dense d'un ancien site pétrochimique rend possible une analyse exploratoire détaillée des données. Cette étape préalable, indispensable à toute procédure d'estimation, a pour objet d'identifier la variabilité spatiale des concentrations et de la quantifier, notamment à l'aide du variogramme.

Pour ce site complexe, une étude de sensibilité montre l'apport d'une modélisation géostatistique détaillée, notamment pour tenir compte de l'hétérogénéité de la répartition des concentrations sur le site. La technique de validation croisée permet ensuite d'évaluer les incertitudes entachant inévitablement les estimations. Nous plaçant dans le cas particulier d'une sélection «ponctuelle», nous comparons alors les « résultats effectifs » aux prévisions de concentration des sols sélectionnés, et explicitons ainsi l'une des causes des écarts fréquemment constatés entre le projet de dépollution et les résultats effectifs. Une méthode de calcul, rapide et rigoureuse, des quantiles de la concentration ou de la proportion de valeurs dépassant un seuil fixé est enfin rappelée.
Seules les concentrations sont examinées ici ; d'autres critères interviennent pour qualifier l'aptitude de la pollution au transfert vers différentes cibles.

L'ancien site pétrochimique étudié couvre une aire d'environ $1700 \times 1400 \mathrm{~m}$. La variable étudiée est la concentration en hydrocarbures totaux d'échantillons de sols, notée HC sur les figures. À la demande du fournisseur des données, les unités de concentration ne sont pas précisées.

\section{VARIABILITÉ SPATIALE DES CONCENTRATIONS}

La représentativité spatiale d'un échantillon dépend de nombreux facteurs, comme les propriétés du sol ou la technique d'échantillonnage et d'analyse (y compris le conditionnement et le transport des échantillons). En pratique, «la zone d'influence » attribuée à un échantillon est encore parfois directement déduite du plan d'échantillonnage, sans référence à la variabilité spatiale des concentrations.

L'examen approfondi des données disponibles, dont l'acquisition est pourtant onéreuse, reste une étape souvent négligée. Nous détaillons donc un exemple d'analyse variographique exploratoire, pour montrer qu'elle peut fournir une description détaillée de la répartition et du niveau des concentrations, permettant ainsi une meilleure compréhension de l'agencement de la pollution sur le site.

\subsection{Implantation des données}

Le schéma d'échantillonnage comporte 194 points de mesures. Justifié par l'historique du site, l'échantillonnage

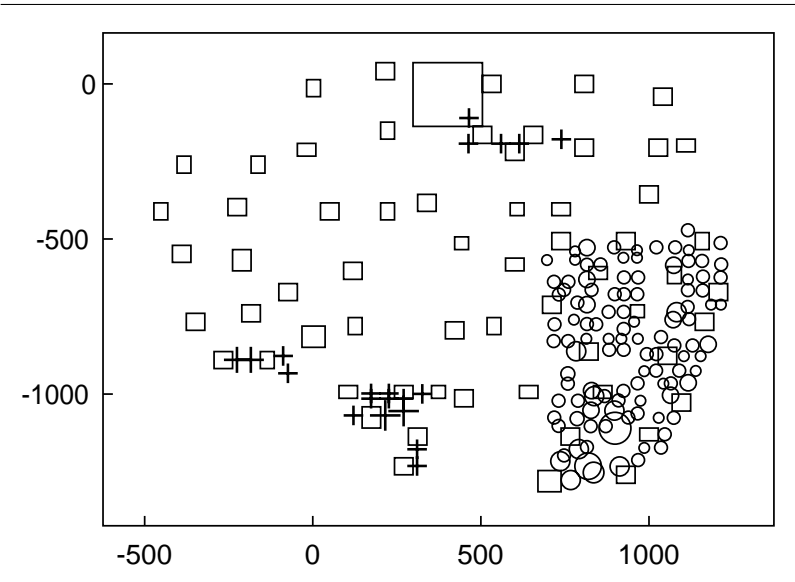

Figure 1

Implantation des prélèvements. Carrés : pseudo-grille ; cercles : autres échantillons de la zone des UP ; croix : autres échantillons. La dimension des symboles est proportionnelle à la concentration en profondeur. L'unité de longueur est le mètre. Location of the sample points. Squares: quasi regular mesh; circles: other sample points on the UP; crosses: other sample points. The dimension of the symbols is proportional to the depth total hydrocarbon concentration. The length unit is the meter. 
est resserré à environ $35 \mathrm{~m}$ sur les anciennes unités de production (notées UP dans la suite), où il présente quelques lacunes correspondant à des infrastructures (fig. 1). Pour éviter que cette densité d'échantillonnage variable ne perturbe les statistiques, 63 données, réparties à peu près régulièrement sur tout le site, ont été sélectionnées pour constituer une «pseudo-grille» à maille d'environ $150 \mathrm{~m}$. La pseudo-grille recoupe localement la zone des UP.

\subsection{Concentration en surface et en profondeur}

En tout point expérimental, deux prélèvements ont été effectués : l'un dans les niveaux superficiels, qualifié de «surface»; l'autre en «profondeur», implanté au niveau de la nappe. Pour chaque niveau, nous supposons les échantillons de même longueur et de même volume, et prélevés à la même profondeur.

Les statistiques élémentaires pour la pseudo-grille indiquent, pour l'ensemble du site, une concentration plus forte en profondeur qu'en surface (tableau 1). L'écart type ainsi que le coefficient de variation (le rapport de l'écart type à la moyenne, qui mesure la dispersion relative des valeurs autour de leur moyenne) sont élevés pour les concentrations mesurées en profondeur, du fait de la très forte valeur maximale de la concentration maximale. Celle-ci est sans influence sur la médiane, environ deux fois plus forte en profondeur qu'en surface.

Sur les UP, la moyenne, la médiane ainsi que les maxima sont analogues en surface et en profondeur.
TABLEAU 1

Statistiques de la concentration en hydrocarbures totaux Statistics of the total hydrocarbon concentration

\begin{tabular}{l|c|c|c|c}
\hline & \multicolumn{2}{|c|}{ Pseudo-grille } & \multicolumn{2}{c}{ UP } \\
\hline & Surface & Profond. & Surface & Profond. \\
\hline $\mathrm{Nb}$. & 63 & 63 & 131 & 128 \\
Min. & 1 & 19 & 1 & 1 \\
Max. & 15934 & 53412 & 32004 & 31408 \\
Moy. & 900 & 1807 & 1532 & 1681 \\
Q50 & 121 & 267 & 247 & 207 \\
$\sigma$ & 2587 & 6695 & 4139 & 3952 \\
б/moy. & 2,87 & 3,71 & 2,70 & 2,35 \\
\hline
\end{tabular}

$\mathrm{Nb}$ : nombre de mesures ; min. : minimum ; max. : maximum ; moy. : moyenne ; Q50: médiane; $\sigma$ : écart type. Le rapport de l'écart type à la moyenne est le coefficient de variation.

$N b$ : number of measurements; min.: minimum; max.: maximum; moy.: mean, Q50: median, $\sigma$ : standard deviation. The ratio of standard deviation and mean is the coefficient of variation.

En résumé, la concentration est supérieure en profondeur sur la pseudo-grille, et localement plus forte en surface sur les UP. Cette observation justifie le resserrement local de la maille sur ces UP, guidé par la connaissance du site. Sur les UP, les statistiques de concentration, analogues en surface et en profondeur, pourraient indiquer un remaniement local du terrain, ayant mélangé les sols de surface avec ceux en profondeur.

La concentration en surface ainsi que la répartition croisée des concentrations en surface et en profondeur ne sont donc pas stationnaires sur l'ensemble du site.
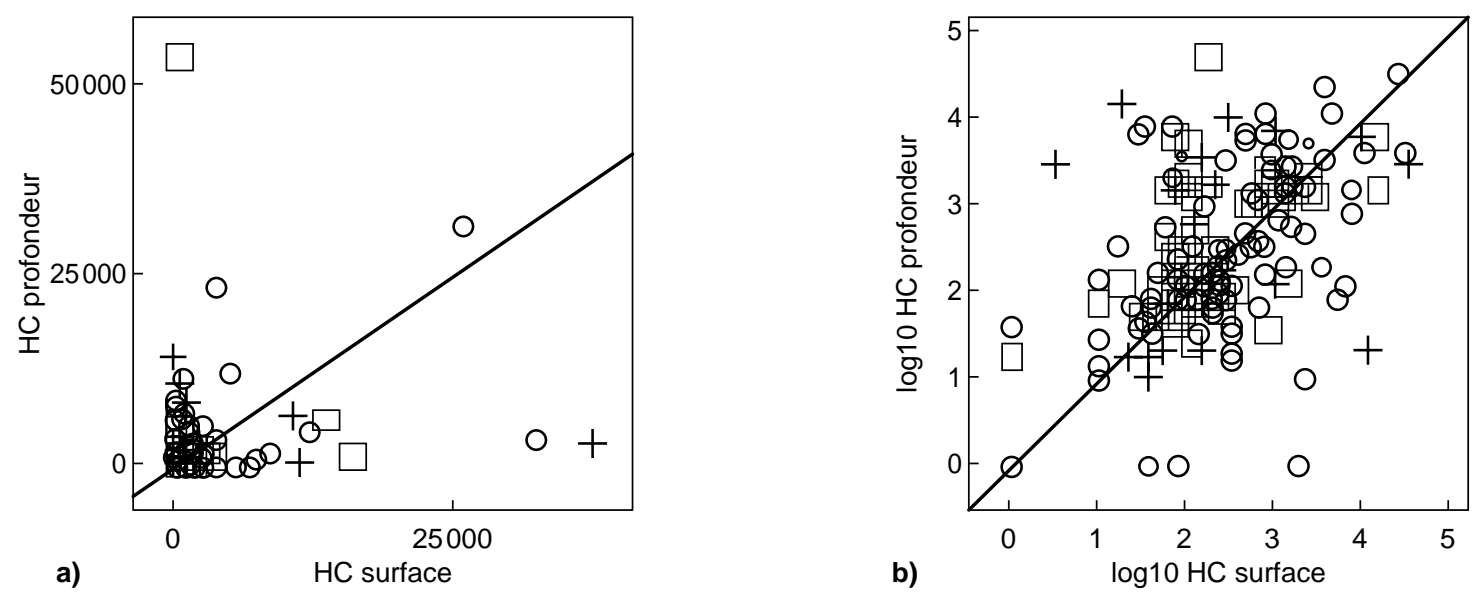

Figure 2

Nuage de corrélation des concentrations en hydrocarbures totaux (a) et de leurs logarithmes décimaux (b), pour les mesures en surface (abscisse) et en profondeur (ordonnée). Mêmes symboles qu'en figure 1. La première bissectrice est reportée. Coefficient de corrélation des concentrations : 0,23 et des logarithmes : 0,51 .

Correlation plot of the total hydrocarbon concentrations (a) and of the decimal logarithms $(b)$ of the measurements near the surface (abscissa) and in depth (ordinate). Same symbols as in Figure 1. The first bisector is shown. Correlation coefficient of the concentrations: 0.23 and of their logarithms: 0.51 . 
Les concentrations en surface et en profondeur ne présentent quasiment pas de corrélation, ce que confirme le nuage des logarithmes, fortement dispersé : à une concentration en surface voisine de 100 peut correspondre une forte valeur en profondeur, et réciproquement, les fortes concentrations en profondeur sont associées à des concentrations en surface variables sur plus de deux ordres de grandeur (fig. 2). Ce constat, valable également pour d'autres sites (voir Jeannée, 2001), met en cause la pratique consistant, notamment lors d'une dépollution par couches de profondeur croissante, à arrêter en un point du site l'échantillonnage verticalement dès la première concentration faible rencontrée.

\subsection{Hétérogénéité du site}

D'après les statistiques de la pseudo-grille, les concentrations en profondeur couvrent un grand intervalle de valeurs, de 19 à 53000 . Le coefficient de variation élevé (égal à 3,71) reflète cette forte dispersion. De ce fait, l'histogramme des concentrations apparait peu lisible, contrairement à celui des logarithmes, qui présente une évidente bimodalité (fig. 3, où la valeur maximale est supprimée de l'histogramme des concentrations, mais conservée sur celui des logarithmes). Les deux modes disjoints se situent respectivement autour de 75 et de 1500, avec une séparation autour de 800 ; la bimodalité se retrouve sur les UP. Une légère bimodalité est également admissible sur l'histogramme des concentrations en surface, pour lequel les deux modes ne sont plus disjoints mais se recouvrent largement.

Dans la suite, nous examinons les concentrations des seuls échantillons prélevés en profondeur. Le mode des fortes concentrations se localise principalement sur une partie des UP, ainsi qu'en périphérie du site (fig. 3).

Pour des distributions présentant une forte variance, il peut être intéressant de travailler avec le logarithme des concentrations, au moins pour affiner l'étude variographique. Toutefois, nous ne présentons pas l'estimation dans le cadre lognormal, car le «krigeage lognormal» repose sur des hypothèses strictes de stationnarité et de multinormalité des transformées logarithmiques, qui ne sont pas vérifiées ici (Chilès et Delfiner, 1999; Prechtel, 1998).

\section{MODÉLISATION DE LA BIMODALITÉ}

La bimodalité de l'histogramme indique la présence de deux populations, statistiquement différentes, de concentration dite respectivement forte (supérieure à 800) ou faible (inférieure à 800). Spatialement, il n'apparaît pas de frontière délimitant avec netteté l'implantation de ces deux populations (fig. 3).

\subsection{Trois modèles de distribution spatiale}

Est-il réellement nécessaire de tenir compte de l'hétérogénéité du site, mise en évidence par la bimodalité de
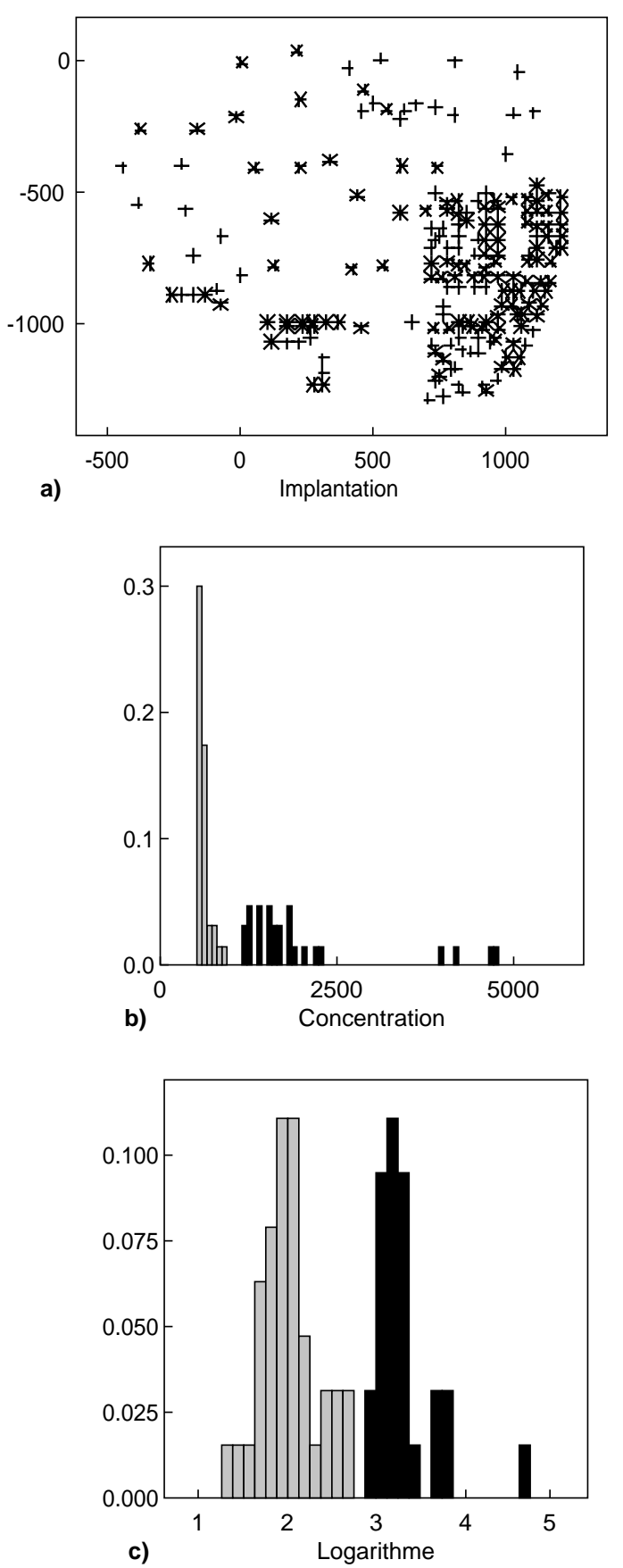

Figure 3

a) implantation des deux modes et histogramme des concentrations en profondeur pour la pseudo-grille; b) concentrations hors la valeur maximum; c) logarithme décimal. Étoiles : mode des concentrations inférieures à 800 ; croix : mode des concentrations supérieures à 800 . L'unité de longueur est le mètre.

a) location of the two modes and histogram of the concentration in depth for the quasi regular mesh; b) concentrations, except the maximum value; c) decimal logarithm. Stars: mode of the concentrations inferior to 800, crosses: mode of the concentrations superior to 800 . The length unit is the meter. 
l'histogramme, pour cartographier les concentrations ? Pour vérifier l'intérêt d'une modélisation détaillée de la variabilité spatiale, nous calons trois modèles de complexité croissante, qui seront ensuite comparés numériquement suivant des critères de précision de l'estimation.

- Le site est traité dans son ensemble, ce qui revient à supposer les concentrations statistiquement homogènes.

- Le site est partagé en deux classes non connexes, qualifiées de «forte» ou «faible», associées respectivement aux deux modes de l'histogramme, supérieur ou inférieur à 800 . Pour estimer la concentration en un point non informé (aux nœuds d'une grille régulière par exemple), il est nécessaire de modéliser l'implantation spatiale de ces deux classes.

- Dans un modèle «intermédiaire », le site est divisé en deux ensembles, délimités empiriquement par lissage du contour des classes précédemment définies suivant les deux modes (fig. 4). Sur les UP, la délimitation suit les lacunes du plan d'échantillonnage, correspondant à une voie; ce découpage s'écarte ainsi des contours irréguliers des classes associées aux modes de l'histogramme. Relativement aux données disponibles, ce découpage est établi plus grossièrement sur les UP que sur le reste du site. Les deux ensembles ainsi délimités, notés respectivement mixte-fort et mixte-faible, présentent chacun une distribution approximativement unimodale (fig. 5).

Pour d'autres sites, une partition en zones approximativement homogènes devrait s'appuyer sur les connaissances disponibles, parmi lesquelles l'historique du site. Pour être fiable, cet historique doit être suffisamment informé, en particulier sur les événements survenus lors de la fermeture du site, ou ultérieurement. En pratique, il est difficile de s'assurer que tel est effectivement le cas.

Les statistiques élémentaires pour ces deux partitions sont reportées au tableau 2, les statistiques du site traité de façon

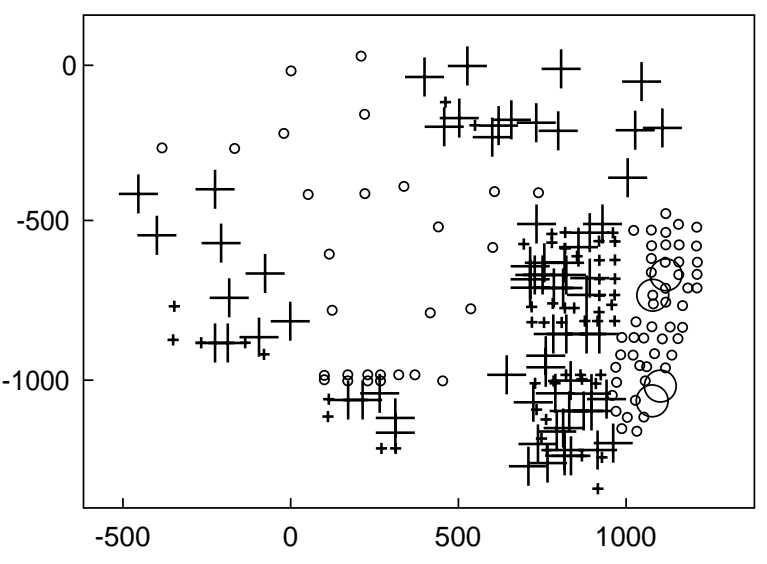

Figure 4

Délimitation des zones mixte-faible (cercles) et mixte-fort (croix). La dimension des symboles indique si la concentration est inférieure ou supérieure à 800 . L'unité de longueur est le mètre.

Location of the mixed-weak zone (circles) and the mixedhogh zone (crosses). The dimension of the symbols indicates if the concentration is inferior or superior to 800 . The length unit is the meter.

homogène figurant au tableau 1. Malgré le nombre important de données initiales, après partition suivant les classes de valeur et l'implantation des mesures, les statistiques portent sur un nombre parfois réduit de données. Pour l'interprétation des écarts types, il faut tenir compte du nombre de données mais également de leur implantation spatiale: les variances expérimentales, qui ont le sens de variances de dispersion, dépendent en effet du «champ » (Matheron, 1970).

Logiquement, les statistiques sont plus fortement contrastées pour la partition suivant les modes de l'histogramme que pour les classes mixtes, ces dernières incluant respectivement
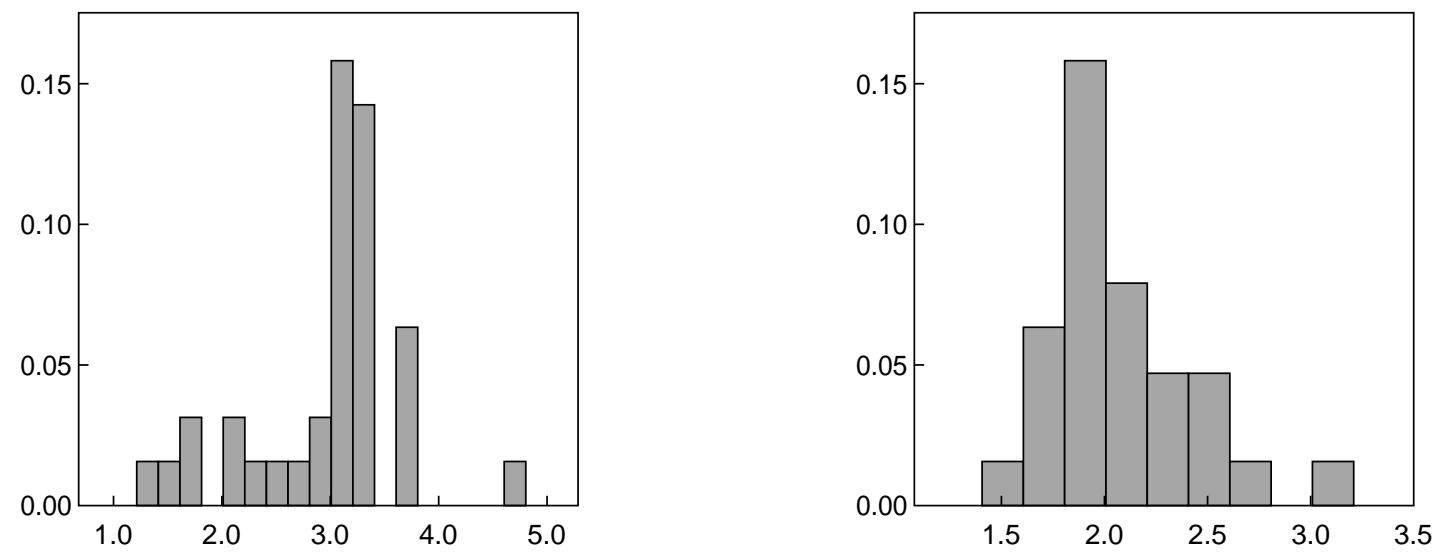

Figure 5

Histogramme du logarithme décimal de la concentration pour la pseudo-grille. Gauche : mixte-fort ; droite : mixte-faible.

Histogram of the decimal logarithm of the concentration on ther quasi regular mesh. Left: mixed-high; right: mixed-weak. 
quelques fortes concentrations dans la classe mixte-faible et quelques faibles concentrations dans la classe mixte-fort (voir le maximum et le minimum respectifs, tableau 2). Par rapport à l'ensemble du site, caractérisé par les statistiques de la pseudo-grille, les UP présentent une moyenne et une médiane supérieures pour le mode des valeurs fortes. Pour le mode des valeurs faibles, les statistiques sont analogues pour la pseudo-grille et les UP. Le contraste entre les UP et le reste du site apparaît moins clair pour le découpage en classes mixtes : pour la classe mixte-fort, la médiane et la moyenne sont inférieures pour les UP, alors que pour la classe mixtefaible, la moyenne est deux fois plus forte pour les UP, les médianes étant identiques pour les UP et le reste du site. À noter que sur les UP, la médiane de la classe mixte-fort (873) est proche de la limite entre les modes (800).

\section{TABLEAU 2}

Statistiques par classe

Statistics according to concentration classe

\begin{tabular}{l|c|c|c|c}
\hline & \multicolumn{2}{|c|}{$\mathrm{HC}>800$} & \multicolumn{2}{c}{ HC $<800$} \\
\hline & $\mathrm{psd}$ & $\mathrm{UP}$ & $\mathrm{psd}$ & $\mathrm{UP}$ \\
\hline $\mathrm{Nb}$ & 27 & 43 & 36 & 85 \\
Min. & 950 & 873 & 19 & 1 \\
Max. & 53412 & 31408 & 530 & 670 \\
Q50 & 1600 & 2880 & 91 & 89 \\
Moy. & 4038 & 4708 & 134 & 149 \\
$\sigma$ & 9790 & 5713 & 115 & 151 \\
\hline \multirow{2}{*}{ Mixte-fort } & Mixte-faible \\
\hline Nb & psd & UP & psd & UP \\
\hline Min. & 35 & 77 & 28 & 51 \\
Max. & 53412 & 19 & 27 & 10. \\
Q50 & 1400 & 873 & 1488 & 3592 \\
Moy. & 3111 & 2563 & 177 & 89 \\
$\sigma$ & 8763 & 4857 & 271 & 789 \\
\hline
\end{tabular}

$\mathrm{HC}$ : concentration en hydrocarbures totaux; $\mathrm{Nb}:$ nombre de mesures ; min. : minimum ; max. : maximum ; moy. : moyenne ; Q50 : médiane ; $\sigma$ : écart type ; psd : pseudo-grille; UP : unités de production.

HC: total hydrocarbon concentration; Nb: number of measurements; min.: minimum; max.: maximum; moy.: mean; Q50: median; $\sigma:$ standard deviation; $p$ sd: quasi regular mesh; UP: former production units.

Ces résultats sont cohérents avec un « mélange » fin entre fortes et faibles concentrations sur les UP, évoqué lors de la comparaison des valeurs en surface et en profondeur. La régularité des contours des classes mixtes étant analogue pour l'ensemble du site et pour les UP, les classes mixtes respectivement forte ou faible incluent sur ces UP proportionnellement plus de concentrations appartenant à l'autre mode. Le tracé plus irrégulier des frontières, rendu possible par l'échantillonnage resserré sur les UP, aurait permis de délimiter plus finement les classes mixtes.

L'irrégularité du contour des zones de fortes concentrations est ainsi mise en évidence sur les UP grâce à l'échantillonnage resserré. Qu'en est-il sur le reste du site ? L'échantillonnage actuel ne permet pas de répondre à cette question.

Remarque : sur la pseudo-grille, le contraste entre le mode des valeurs fortes et celui des valeurs faibles est voisin de 30 pour la moyenne et de 18 pour la médiane. Pour les classes mixtes, ces deux rapports sont proches, avec respectivement 17,5 et 15 . Un seul critère, comme la moyenne ou la médiane, ne suffit donc pas à synthétiser les contrastes de concentration entre les différentes classes ; la dispersion des valeurs dans les classes est également importante.

\subsection{Calcul et ajustement des variogrammes}

Lorsque le coefficient de variation est élevé, le variogramme des logarithmes apparaît souvent moins erratique que celui des concentrations. Le passage au logarithme est alors utile pour préciser la stationnarité ou les anisotropies, ou pour vérifier le caractère «pépitique» du variogramme. Dans le cas présent, ce calcul n'améliore pas systématiquement l'allure des variogrammes expérimentaux. Malgré l'importante dispersion des concentrations, nous présentons donc le calcul et l'ajustement du variogramme de la concentration, le variogramme des logarithmes étant utilisé pour préciser quelques particularités.

Les variogrammes directionnels expérimentaux n'indiquant pas d'anisotropie nette, un modèle de variogramme isotrope est ajusté sur les variogrammes expérimentaux omnidirectionnels. L'ajustement des variogrammes pour la pseudo-grille (fig. 6), récapitulé au tableau 3, s'effectue à une affinité près sur le palier de la structure à petite portée, celle-ci étant calée d'après le variogramme local des UP. Les structures à grande portée indiquent une nonstationnarité à l'échelle du site, la portée ajustée restant approximative.

\section{TABLEAU 3}

Variogramme de la concentration par classe ; ajustement pour la pseudo-grille

Variogram model of the concentration per classe; fitting for the quasi regular mesh

\begin{tabular}{l|l}
\hline Homogène & $10^{6}[15+4,5 \mathrm{sph}(100 \mathrm{~m})+45 \mathrm{sph}(1500 \mathrm{~m})]$ \\
\hline $\mathrm{HC}>800$ & $10^{7}[7+5 \mathrm{sph}(1000 \mathrm{~m})]$ \\
\hline $\mathrm{HC}<800$ & $10^{3}[8,5+3,5 \mathrm{sph}(150 \mathrm{~m})+3,0 \mathrm{sph}(500 \mathrm{~m})]$ \\
\hline Mixte-fort & $10^{7}[3,0+1,8 \mathrm{sph}(100 \mathrm{~m})+2,4 \mathrm{sph}(700 \mathrm{~m})]$ \\
\hline Mixte-faible & $10^{3}[7,5+12,5 \mathrm{sph}(150 \mathrm{~m})+65 \mathrm{sph}(1200 \mathrm{~m})]$ \\
\hline
\end{tabular}

Le modèle ajusté est la somme d'un effet de pépite et d'un ou de deux schémas sphériques. sph (a) désigne un variogramme sphérique de palier 1 et de portée a (Chilès et Delfiner, 1999).

The fitted model consists of the sum of a nugget effect and one or two spherical schemes. sph (a) denotes a spherical model with sill1 and range a (Chilès et Delfiner, 1999). 


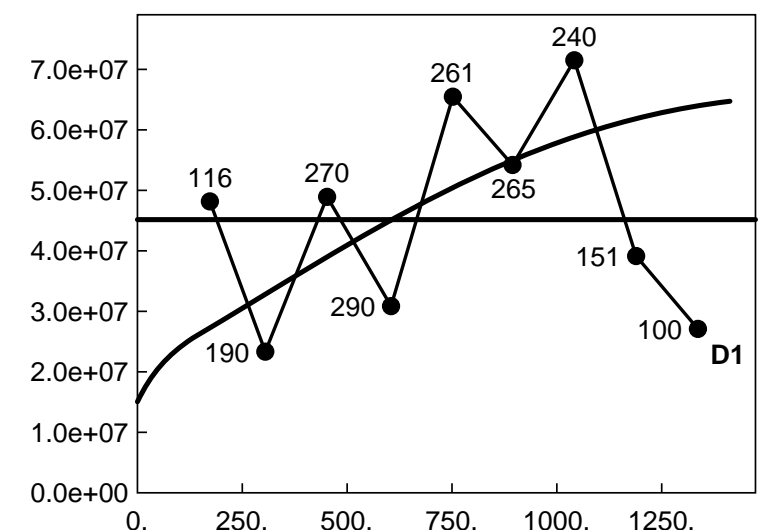

a)

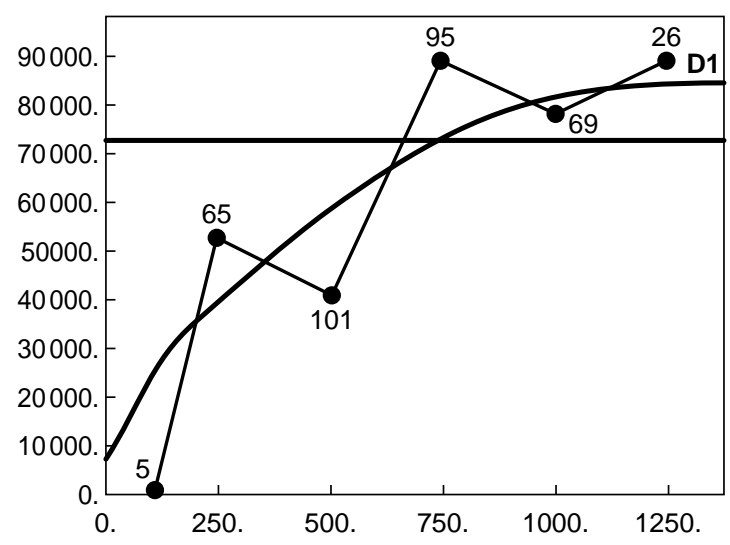

c)

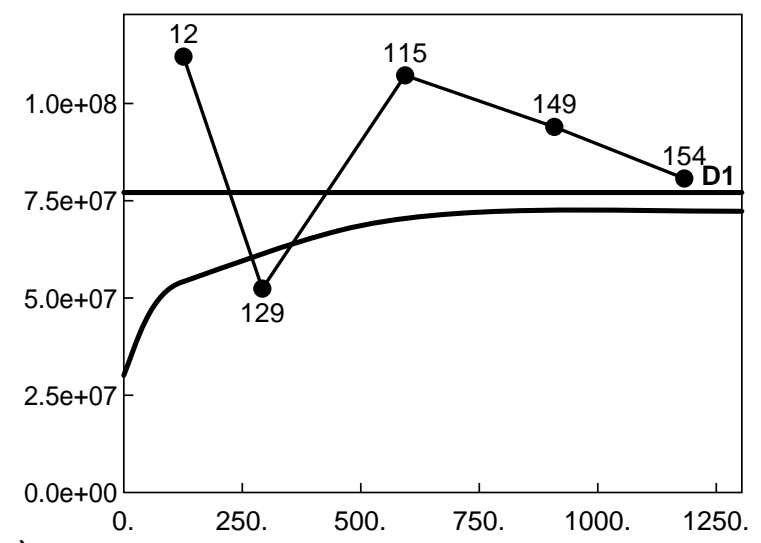

e)

Figure 6

Variogramme de la concentration et ajustement pour la pseudo-grille. Les chiffres indiquent le nombre de couples de points pour chaque pas du variogramme expérimental. a) population «homogène» : pseudo-grille; b) population «homogène »: UP ; c) classe mixte-faible : pseudo-grille ; d) classe mixte-faible : UP ; e) classe mixte-fort ; pseudo-grille ; f) classe mixte-fort ; UP.

Variogram of the concentration and fitting for the quasi regular mesh. The number of couples of points for each step of the experimental variogram is reported. a) "homogeneous population": quasi regular mesh; b) "homogeneous population": UP; c) mixed weak class: quasi regular mesh; d) mixed-weak class: UP; e) mixed-high class: quasi regular mesh; f) mixed-high class: UP.

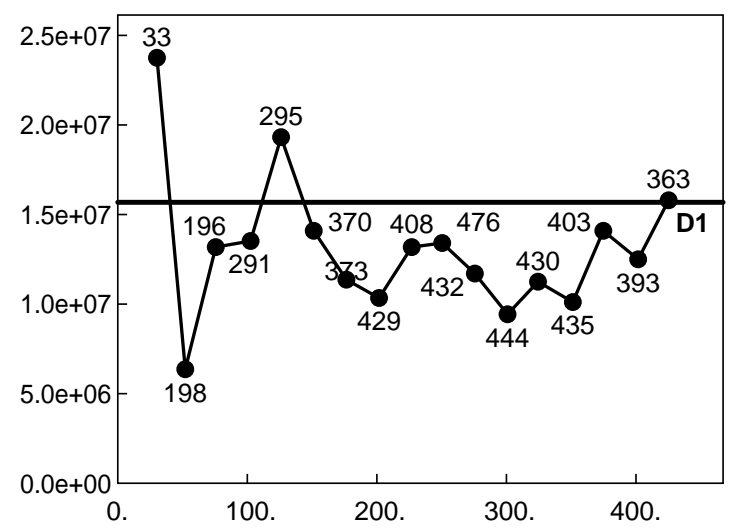

b)
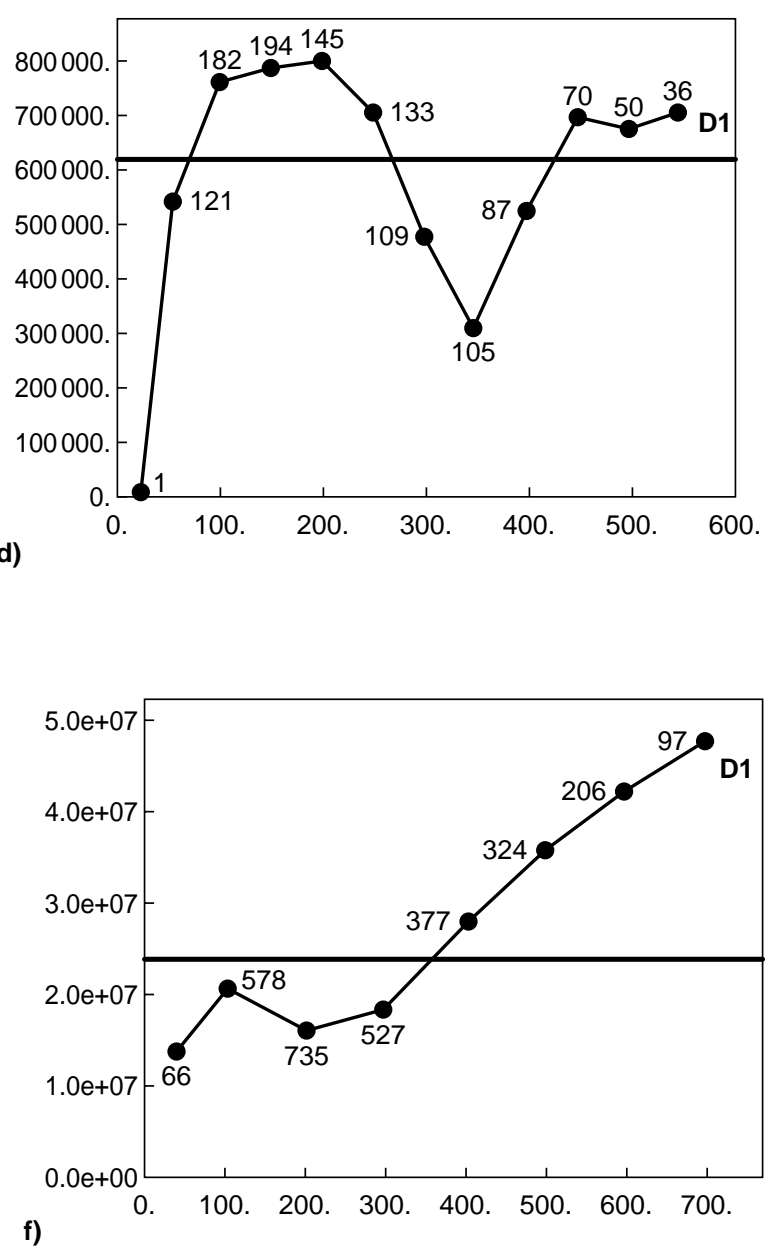
Les principales observations sur les variogrammes expérimentaux sont les suivantes :

- Pour chacun des deux modes, l'effet de pépite est prépondérant dans le palier total : à l'intérieur des classes, la structure spatiale des concentrations est médiocre, en particulier à petite distance (les variogrammes correspondants ne sont pas présentés). Le variogramme de la population complète dépend donc principalement de la localisation relative de ces deux classes.

- Pour la classe mixte-faible, le palier du variogramme est environ dix fois plus faible pour la pseudo-grille que pour les UP, ce qui est cohérent avec la variance et la moyenne, supérieures sur les UP. Pour la pseudo-grille ce variogramme présente une structure spatiale bien marquée aux distances intermédiaires, alors qu'il est quasi «pépitique » dans la zone des UP. On observe l'inverse pour la classe mixte-fort : le variogramme expérimental apparaît erratique sur la pseudo-grille, et structuré sur les unités de production.

- Pour la population complète, l'effet de pépite est important relativement au palier de la structure à petite portée. Ceci est cohérent avec le variogramme des classes de fortes concentrations, dont la variabilité spatiale est prédominante dans l'ensemble de la population.

Un examen détaillé de la zone des UP montre que les sept échantillons de concentration minimale et égale à l'unité sont regroupés et affectés à la classe mixte-fort (fig. 7). Localement, ces concentrations quasiment nulles voisinent avec des valeurs supérieures à 1500 . Calculé sur une petite zone entourant les sept points précédents, le variogramme expérimental du logarithme de la concentration présente un effet de pépite pur.

Supprimer cette petite zone divise par deux le palier du variogramme des logarithmes de la classe mixte-fort sur les UP. Ce variogramme conserve de fortes valeurs à petite distance, mais présente une structure spatiale d'une portée de $100 \mathrm{~m}$ environ (fig. 7). Cette valeur de la portée n'est pas retenue pour l'ajustement du variogramme de la concentration, le variogramme du logarithme n'admettant ni les mêmes paliers relatifs, ni les mêmes portées pratiques que celui de la variable initiale (Matheron, 1989).

En résumé, sur les anciennes UP, une partie de la variabilité dans la zone mixte-fort provient d'une zone très localisée, dans laquelle sont mélangées les concentrations très faibles à fortes. Comme de forts contrastes se retrouvent également ailleurs entre échantillons distants de moins $30 \mathrm{~m}$, la classe mixte-fort dans les UP est, par la suite, traitée dans son ensemble - le variogramme modélisé indiquant une forte variabilité à petite distance.

L'absence locale de corrélation spatiale sur une petite zone des UP, traduisant un « mélange » très fin de concentrations contrastées, est cohérente avec l'hypothèse d'un remaniement local des sols pollués excavés ayant été remblayés par des sols non pollués. L'historique détaillé du site expliquerait la présence paradoxale de ces quelques concentrations quasi nulles disséminées dans une zone «polluée».

\subsection{Interprétation des variogrammes}

L'ajustement des variogrammes synthétise les observations sur la variabilité spatiale des concentrations. Nous examinons les paliers, l'amplitude de l'effet de pépite, ainsi que les portées.

\subsubsection{Paliers}

Lorsque les concentrations peuvent être supposées stationnaires, le palier du variogramme constitue un indicateur de la dispersion des valeurs, moins sensible à la dimension du champ et à une implantation irrégulière des données que la variance expérimentale (Matheron, 1970). On rappelle que les paliers, ayant la dimension d'une variance, ont comme unité le carré de la concentration.

Les paliers totaux ajustés pour la pseudo-grille suivent la dispersion des concentrations dans les différents ensembles. Les paliers minimaux correspondent aux deux ensembles de concentrations faibles. Le palier pour le mode faible $\left(1,510^{4}\right)$ est inférieur à celui pour la classe mixte-faible $\left(8,510^{4}\right)$, celle-ci comportant quelques concentrations plus élevées. Dans le modèle de site homogène, le variogramme expérimental moyenne différents niveaux d'écarts quadratiques, ce qui fournit un palier intermédiaire, égal à $6,510^{7}$. Le palier ajusté pour la classe mixte-fort est à peine supérieur $\left(7,010^{7}\right.$, mais une valeur supérieure était tout aussi admissible, vu le caractère erratique du variogramme expérimental, fig. 6). Le palier maximum $\left(1,210^{8}\right)$ est ajusté pour le mode des concentrations supérieures à 800 .

Le palier du variogramme intervient en particulier dans le calcul de la variance des erreurs d'estimation. Multiplier le variogramme par une constante, toutes choses égales par ailleurs, laisse inchangée l'estimation et multiplie d'autant la variance de krigeage (Matheron, 1970).

\subsubsection{Effets de pépite}

Les effets de pépite ajustés sont voisins pour les deux ensembles de faibles concentrations $\left(7,510^{3}\right.$ pour la classe mixte-faible, $8,510^{3}$ pour le mode faible). Intermédiaire pour le modèle homogène $\left(1,510^{7}\right)$, l'effet de pépite est élevé pour les classes de fortes concentrations $\left(3,010^{7}\right.$ pour la classe mixte-fort, et maximum avec $7,010^{7}$ pour le mode fort).

L'effet de pépite intègre au moins deux causes de variabilité : la variance de l'erreur d'échantillonnage (acquisition, manutention, mesure, etc.), ainsi que la variabilité spatiale des concentrations pour les distances inférieures aux distances minimales entre points expérimentaux. Les effets de pépite ajustés, supérieurs pour les ensembles de concentrations élevées, sont ici compatibles avec l'hypothèse d'une variance d'erreur de mesure croissante avec la concentration. 

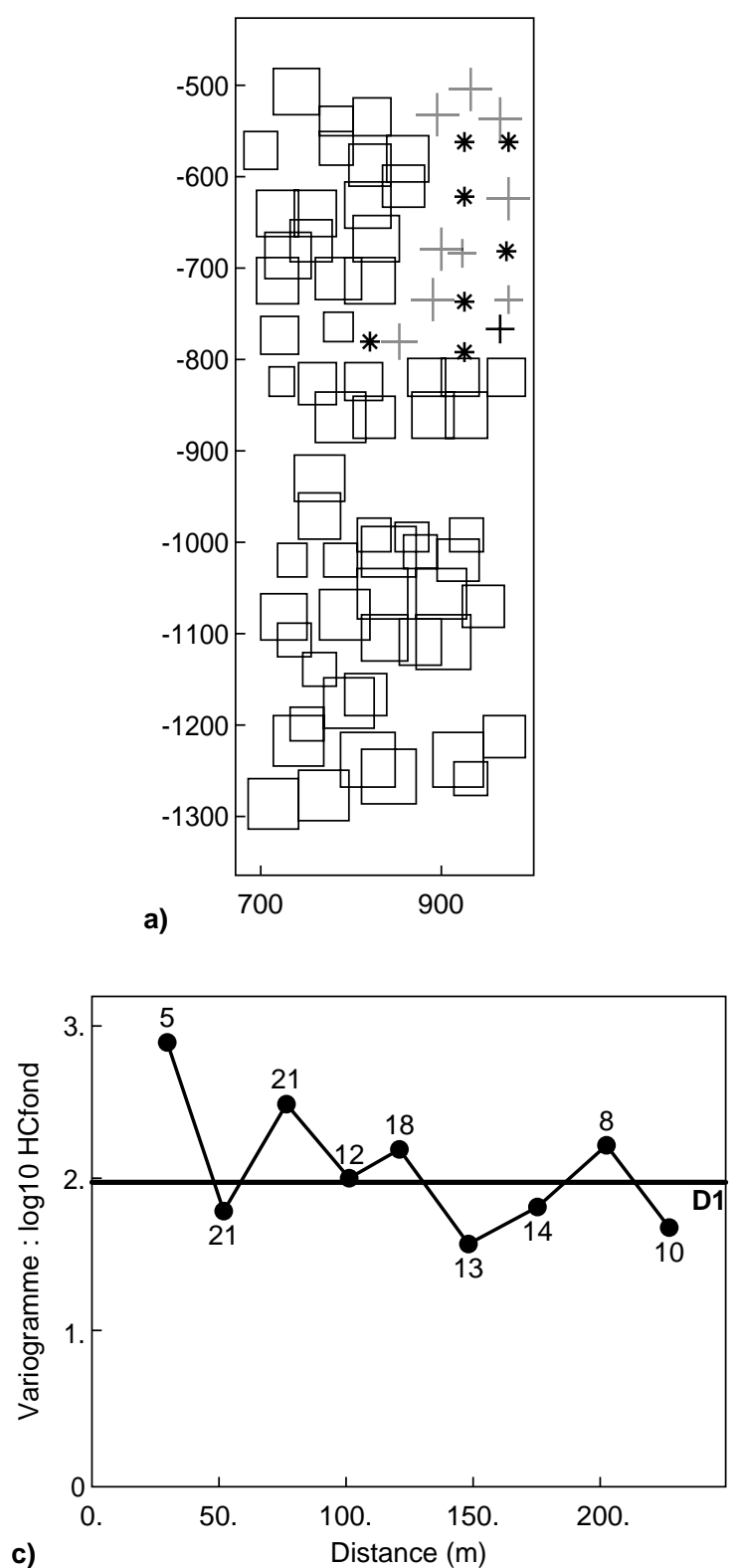
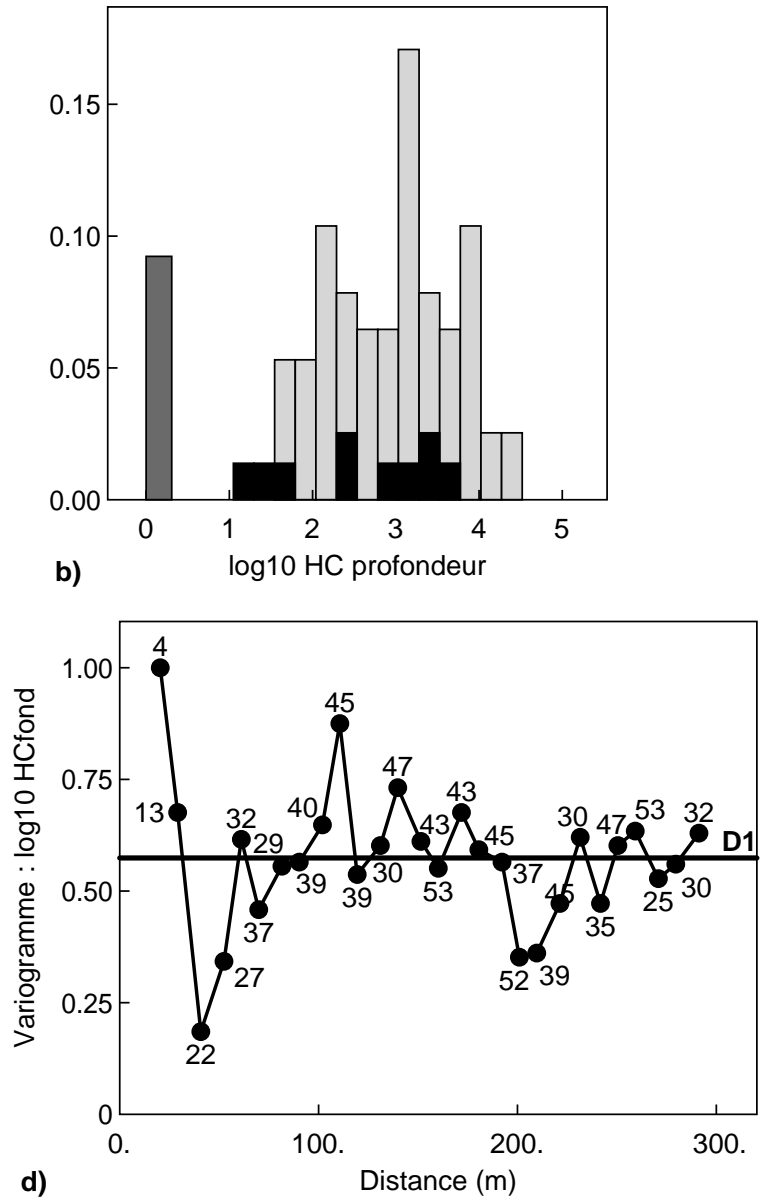

Figure 7

Zone mixte-fort sur les UP. Influence d'une petite zone de fort contraste de concentrations.

a) implantation des concentrations égales à l'unité (étoiles) et délimitation de la petite zone (étoiles et croix); b) histogramme du logarithme décimal des concentrations. Gris foncé : valeurs de la petite zone, égales à l'unité (étoiles). Noir : autres concentrations dans la petite zone (croix) ; c) variogramme du logarithme décimal des concentrations pour la petite zone (étoiles et croix); d) variogramme du logarithme décimal des concentrations sur le complémentaire (carrés).

Mixed high class on the production Units. Influence of a small zone of high concentration contrast.

a) location oh the concentrations equal to unity (stars) and limit of the "small" zone (stars or crosses); b) histogram of the logarithm of the concentrations. Dark grey concentrations equal to the minimum (stars). Black: other concentrations of the small zone (crosses); c) variogram of the logarithm of the concnetration for the small zone (stars and crosses); d) variogram of the logarithm for the complementary concentrations (squares). 


\subsubsection{Portées}

L'ajustement des variogrammes comporte une composante à petite portée (100 ou $150 \mathrm{~m})$, sauf pour le mode supérieur à 800 , composé d'un effet de pépite prépondérant et d'une structure à grande portée par rapport aux dimensions du site. Ceci traduit une non-stationnarité à cette échelle, superposée à une très forte variabilité aux petites distances.

La structure à petite portée, ajustée sur les variogrammes de la zone des UP, serait inaccessible avec un échantillonnage à maille hectométrique, comme c'est le cas pour la pseudo-grille. Le resserrement local de la maille de reconnaissance, aussi bien sur des zones de concentrations fortes que plus faibles, s'avère indispensable pour l'inférence du variogramme.

Contrairement aux paliers, les portées ne semblent présenter aucune variation systématique.

La structure spatiale des différents ensembles varie ainsi sensiblement selon le découpage adopté. La forte composante «pépitique » a pour conséquence une estimation imprécise des concentrations.

\subsection{Validation croisée}

Dans sa version usuelle, la validation croisée consiste à supprimer successivement chacune des données pour l'estimer ensuite par krigeage à partir des autres (Chilès et Delfiner, 1999). Cette technique permet notamment :

- de comparer les performances de différents modèles variographiques, ou de mener une étude de sensibilité des estimations aux paramètres du modèle (voisinage, paliers relatifs, portées, etc.) ;

- d'évaluer l'ordre de grandeur des erreurs pour l'estimation ponctuelle. En effet, la variance de krigeage fournie par le modèle n'est pas directement transposable en intervalle de confiance, et sa validité dépend de la qualité de l'ajustement pour la zone estimée. L'histogramme expérimental des erreurs standardisées (erreur divisée par l'écart type de krigeage) peut permettre de valider l'hypothèse de normalité des erreurs d'estimation.

Dans cette partie, nous comparons par validation croisée la qualité de l'estimation pour les trois modèles précédents.

Le krigeage est effectué en voisinage unique pour le modèle de population homogène; pour la partition par mode, on utilise un voisinage glissant de $500 \mathrm{~m}$ de rayon, seules étant retenues les données appartenant à la classe du point à estimer. Pour le modèle mixte, le voisinage unique avec soit l'ensemble des données, soit les seules données appartenant à la classe du point à estimer, conduisent à des résultats voisins, car ces deux voisinages mélangent des concentrations contrastées.

Pour la partition par mode, chaque point expérimental à réestimer est affecté à son mode. Ceci revient à supposer que la délimitation spatiale des deux modes soit connue de façon exacte. Dans la réalité, en dehors des points expérimentaux, l'implantation des différentes classes est inconnue, et doit être modélisée, ce qui introduit des erreurs d'affectation des points ou des blocs dans les différentes classes. On peut par exemple utiliser les techniques de simulation d'ensembles aléatoires mises en œuvre pour la modélisation des réservoirs hétérogènes, comme la méthode des «gaussiennes seuillées », bien adaptée lorsque l'implantation des classes apparaît fortement erratique (Beucher et al., à paraître). On peut aussi calculer la probabilité d'appartenance à chaque classe, et en déduire une estimation de la concentration, par composition des estimations obtenues pour chacune des classes.

La concentration maximale mesurée, égale à 53412 et environ neuf fois supérieure à la deuxième valeur forte, est largement sous-estimée en validation croisée : 1214 en modèle homogène, 2148 en modèle mixte et 3103 pour la partition par mode. Ce point de concentration maximale accroît très fortement la variance expérimentale des erreurs de validation croisée, et c'est également le cas pour la mesure maximale sur les UP. Les résultats de validation croisée sont donc présentés avec ou sans ces points (tableau 4 et fig. 8); l'erreur est calculée comme la différence entre la concentration mesurée et son estimation.

\section{TABLEAU 4}

Statistiques des erreurs de validation croisée Statistics of the cross validation errors

\begin{tabular}{l|c|c|c|c}
\hline \multirow{2}{*}{} & \multicolumn{2}{|c|}{$\begin{array}{c}\text { psd } \\
\text { (haut : 63 points, } \\
\text { bas : 62 points) }\end{array}$} & \multicolumn{2}{c}{$\begin{array}{c}\text { UP } \\
\text { (haut : } 128 \text { points, } \\
\text { bas : } 127 \text { points) }\end{array}$} \\
\hline \multirow{2}{*}{ Modes 800 } & Moyenne & $\sigma$ & Moyenne & $\sigma$ \\
& -158 & 692 & 14 & 3211 \\
Mixte & -972 & 1941 & -196 & 2184 \\
\hline Homogène & -32 & 752 & -21 & 3613 \\
& -859 & 1790 & -245 & 2591 \\
\hline
\end{tabular}

$1^{\text {re }}$ ligne : toutes données; $2^{\text {e }}$ ligne : suppression de la concentration maximale mesurée.

1st line: all the data. 2nd line: omission of the maximum measured value. Moyenne: mean, $\sigma$ : standard deviation.

Pour le modèle de partition par mode, toutes les estimations pour le mode faible sont inférieures à 265 (la moyenne des estimations pour ce mode se situant autour de 140), et toutes les estimations dans le mode fort sont supérieures à 1490 .

L'influence de la densité d'échantillonnage sur la précision de l'estimation est examinée en confrontant les résultats de validation croisée pour la pseudo-grille à ceux pour les UP. 

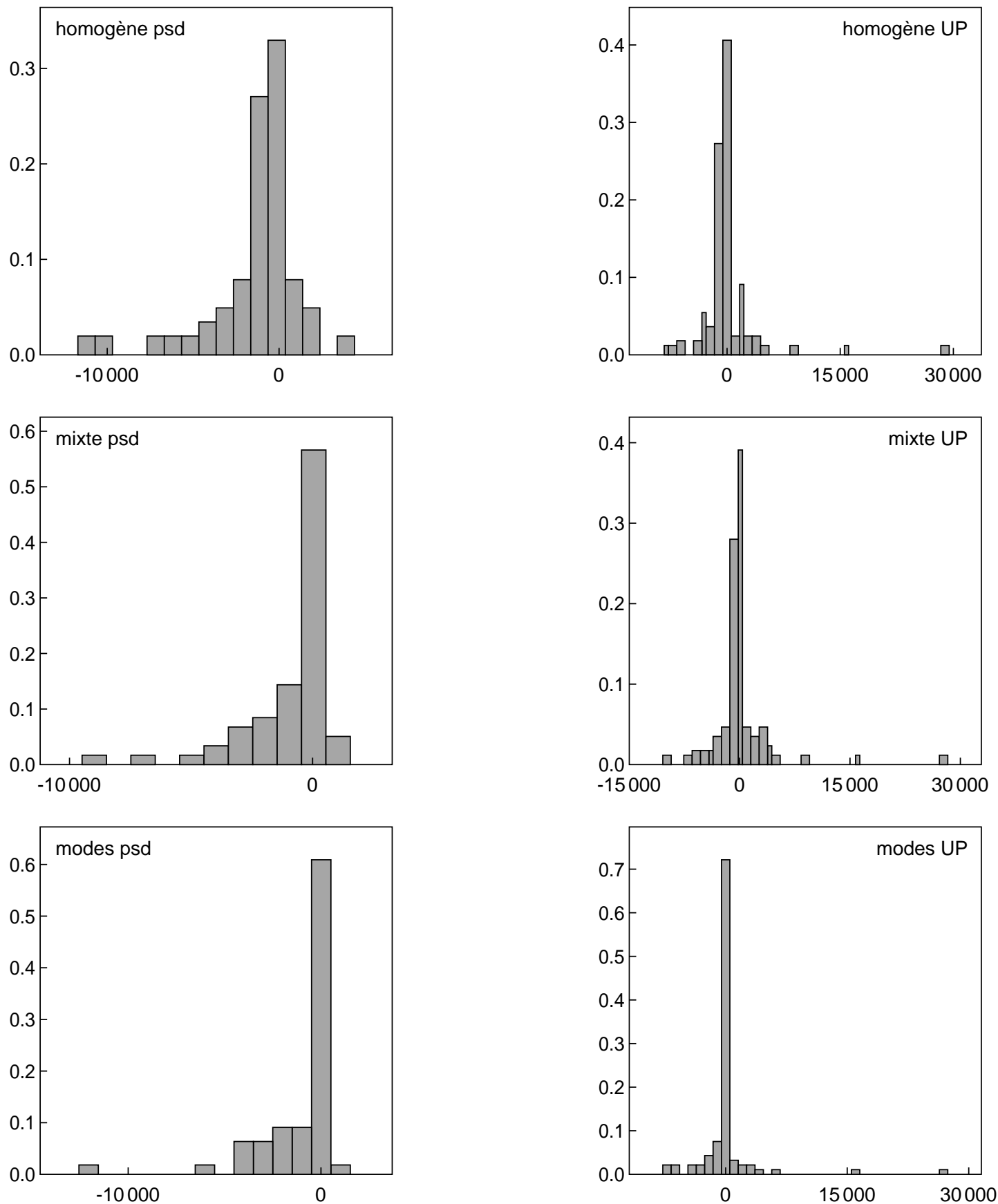

Figure 8

Histogramme des erreurs de validation croisée. Largeur des classes égale à 1000 pour tous les histogrammes. Pour la pseudo-grille régulière (psd), l'erreur correspondant au maximum de la concentration est supprimée.

Histogram of the errors of cross validation. Width of the bars is equal to 1000 for all histograms. For the quasi regular mesh (psd), the errors corresponding to the maximum concentration is omitted.

UP: former production units; "homogene": homogeneous model; "mixte": mixed; "modes": partition by mode. 


\subsubsection{Pseudo-grille}

Pour le découpage par mode, $62 \%$ des erreurs se situent dans l'intervalle [-500,500], contre $57 \%$ pour les classes mixtes et $33 \%$ pour la population supposée homogène (fig. 7). Le complémentaire à $100 \%$ représente les erreurs d'amplitude supérieure à 500, qui dans les trois cas sont en nombre important. Pour la classe $[-100,100]$, les pourcentages respectifs sont de 46, 37 et $16 \%$. La meilleure qualité des estimations pour la séparation en deux classes, notamment suivant les modes, se retrouve sur les statistiques des erreurs (tableau 4), ainsi que sur celles des erreurs relatives (l'erreur divisée par la concentration mesurée). Mais ce résultat fait pour partie illusion: pour le découpage par mode, la classe d'appartenance, connue en validation croisée pour les points expérimentaux, ne l'est plus en un point non échantillonné. L'implantation des différentes classes doit alors être estimée ou simulée, avec inévitablement des erreurs d'affectation.

Le modèle «mixte», dans lequel certaines fortes concentrations sont affectées à la classe mixte-faible tandis que certaines concentrations faibles sont affectées à la classe mixtefort, est assez représentatif des conditions d'estimation réelles. Ce modèle mixte fournit des résultats intermédiaires, clairement meilleurs que ceux obtenus par le modèle homogène.

\subsubsection{UP}

Avec plus de $70 \%$ des erreurs dans la classe [- 500, 500], la partition par mode fournit là encore la meilleure précision ; avec environ $40 \%$ d'erreurs dans cette classe, les résultats sont d'autre part analogues pour les deux autres modèles. Ceci s'explique par le découpage "grossier» des classes mixtes, tandis que la partition par mode opère un découpage fin à l'intérieur de cette zone. Avec une maille dense, l'influence des données du voisinage immédiat augmente au détriment de l'influence du modèle. Sauf en bordure des classes mixtes, les points du voisinage de krigeage les plus proches du point à estimer sont identiques pour le modèle «mixte » et pour le modèle homogène. Une délimitation trop grossière ou inappropriée des classes fortes ou faibles n'apporte pas d'amélioration par rapport au modèle homogène, alors que la partition «exacte» par mode améliore grandement les résultats.

Une erreur d'estimation de 300 suffit à déclasser un point de la concentration intermédiaire, légèrement inférieure à 1100 , vers les concentrations «faibles», inférieures à 800 , tandis que la même erreur d'estimation a des conséquences réduites lorsqu'une concentration de 3500 est estimée à 3200 . C'est pourquoi nous présentons également les statistiques des erreurs relatives (la valeur absolue de l'erreur de validation croisée divisée par la concentration mesurée ; tableau 5).

Pour la partition par mode, les points étant estimés uniquement à partir de données appartenant au même mode, les erreurs relatives sont majoritairement faibles, restant (en valeur absolue) inférieures à l'unité pour plus de $65 \%$ des
TABLEAU 5

Statistiques des erreurs relatives de validation croisée

Statistics of the relative errors of cross validation

\begin{tabular}{l|c|c|c|c}
\hline & \multicolumn{4}{|c}{ Pseudo-grille, 63 points } \\
\hline & Moyenne & Q50 & Q75 & Maximum \\
\hline Modes & 1,1 & 0,66 & 1,56 & 7,4 \\
Mixte & 7,0 & 0,60 & 1,43 & 129,2 \\
Homogène & 12,7 & 1,35 & 15,40 & 122,1 \\
\hline & \multicolumn{4}{|c}{ UP, 128 points } \\
\hline \multirow{4}{*}{ Moyenne } & Q50 & Q75 & Maximum \\
\hline Modes & 11,1 & 0,60 & 1,80 & 253,6 \\
Mixte & 60,3 & 0,89 & 8,86 & 1127,3 \\
Homogène & 53,8 & 1,57 & 10,38 & 1034,7 \\
\hline
\end{tabular}

L'erreur relative est la valeur absolue du rapport de l'erreur de validation croisée à la concentration mesurée. Q50 : médiane, Q75: quantile à $75 \%$.

The relative error is the modulus of the ratio of the cross validation error and the measured concentration. Q50: median, Q75: 75\% quantile.

points de la pseudo-grille. La proportion est analogue avec le modèle mixte, mais chute à $45 \%$ d'erreurs relatives inférieures à l'unité pour le modèle homogène.

Pour ces deux modèles, il apparait quelques très fortes erreurs relatives, supérieures à 100 . Il s'agit notamment des concentrations égales à l'unité, identifiées dans la petite zone «pépitique » de la classe mixte-fort sur les UP, ainsi que de concentrations intermédiaires localisées sur le pourtour du site, et réestimées à l'aide de quelques fortes concentrations situées à proximité.

Dans la suite, nous prenons comme référence le modèle mixte, pour lequel la classe de chaque point est déterminée de façon approchée par le découpage du site. La délimitation approximative des classes de concentrations «fortes » ou «faibles» fournit pour la pseudo-grille des résultats de validation croisée nettement meilleurs que pour le modèle homogène, tandis que sur les UP, ces deux modèles conduisent à des résultats équivalents.

Un modèle bien adapté globalement sur l'ensemble du site ne le reste pas forcément localement. La structure spatiale peut varier sur le site, comme le montre la petite zone «pépitique » identifiée; en pratique, la délimitation des zones de concentration forte ou faible pourrait être affinée là où les données sont localement plus nombreuses.

Remarque : on montre facilement qu'en présence d'erreurs de mesure, l'erreur de validation croisée est la somme de deux termes : l'erreur d'estimation de la concentration et l'erreur de mesure. Pour des erreurs de mesure additives, supposées non corrélées à la concentration, la variance de l'erreur de validation croisée est alors la somme des variances de chacun des termes. Des calculs simples de géostatistique linéaire permettent d'évaluer chacune de ces deux variances. Une forte variance d'erreurs de mesure complique ainsi l'analyse des résultats de validation croisée, la variance 
expérimentale des erreurs n'étant plus directement comparable à la variance d'estimation associée.

\section{KRIGEAGE DE LA MOYENNE PAR BLOC}

Pour «kriger» la concentration, il faut préciser la dimension des blocs (le support) à estimer. Quels peuvent être les critères de ce choix?

En présence d'une forte variabilité spatiale des concentrations à petite distance, connaître parfaitement la concentration en tout point ne constituerait pas une information facilement utilisable: on observerait une succession erratique de pics locaux plus ou moins importants. La moyenne de la concentration calculée, par exemple, sur des blocs d'une dizaine de mètres de côté (dans le plan) fournirait une carte plus lisible, faisant mieux apparaître les zones de concentration en moyenne plus ou moins fortes.

Dans la pratique, les concentrations n'étant pas mesurées en tout point, on ne dispose en dehors des points expérimentaux que d'une estimation de la concentration. Pour un estimateur comme le krigeage, la carte des concentrations estimées est d'autant plus lisse que l'estimation est peu précise (Matheron, 1970). Cela signifie qu'en présence d'un fort effet de pépite, ou à «grande distance» des points expérimentaux, l'estimation varie peu entre deux points voisins. Pour éviter d'interpréter la carte régulière ainsi obtenue comme la réalité, il est préférable d'estimer directement la moyenne de la concentration sur des blocs dont les dimensions sont du même ordre de grandeur que les distances entre points expérimentaux. Ces dimensions peuvent aussi être fixées par les contraintes de la gestion du site.

L'écart type ou l'écart type relatif (l'écart type divisé par la concentration estimée) de l'erreur de krigeage permet de supprimer de la cartographie des concentrations les endroits pour lesquels l'imprécision est trop importante.

L'épaisseur de sol échantillonnée étant supposée constante sur le site, l'estimation s'effectue dans le plan, pour des blocs de cette épaisseur.

Sur les UP, la «maille » d'échantillonnage est d'environ 30 à $40 \mathrm{~m}$, en dehors des lacunes. Pour l'ensemble du site, une grille d'estimation de $25 \mathrm{~m}$ de côté a été retenue. En dehors des UP, cette dimension réduite des blocs estimés accroît de façon sensible la variance de krigeage pour les blocs un peu éloignés des points expérimentaux. Dans le cas présent, la dimension des blocs à estimer pourrait varier sur le site, en fonction de la densité de l'échantillonnage.

Par simplification, le contour des classes a été discrétisé suivant une maille carrée de $25 \mathrm{~m}$ de côté. Le krigeage, effectué en voisinage unique, utilise le variogramme de la classe du bloc à estimer.

La carte de l'écart type d'estimation illustre l'influence du variogramme et de la densité d'information sur la précision (fig. 9). L'influence de la densité de l'échantillonnage est
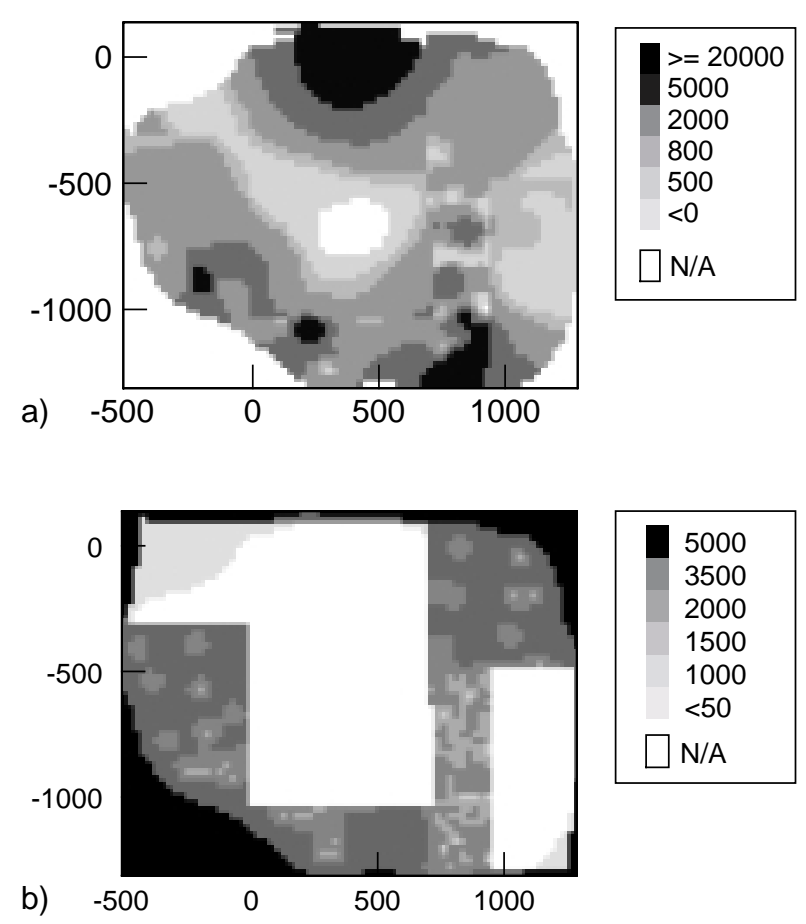

Figure 9

Krigeage (haut) et écart type d'estimation (bas) de la concentration moyenne par bloc. Modèle mixte.

Kriging map (top) and standard deviation of the estimation error (bottom) of the mean concentration in blocks. Mixed model.

évidente dans la zone mixte-fort sur les UP. Elle n'apparaît pas sur la carte des écarts types de krigeage pour la classe mixte-faible : à cause du faible palier du variogramme pour cette classe, l'écart type de krigeage est inférieur à 70 pour tous les blocs de cette classe, alors qu'il est supérieur à 1300 pour tous les blocs affectés à la classe mixte-fort.

Pour la classe mixte-fort, au-delà d'une cinquantaine de mètres autour d'un point expérimental de la grille lâche, l'écart type de krigeage dépasse la valeur 3000 . Bien que le palier n'ait pas été ajusté très précisément, ce fort écart type de krigeage, comparativement aux valeurs de la concentration estimée, montre la précision médiocre des estimations et justifie le resserrement local de la maille de reconnaissance.

Nous visualisons l'estimation pour les blocs dont l'écart type de krigeage est inférieur à 5000.

La présence de la tache de pollution située vers le nord de la carte ainsi que son extension ont été confirmées par un échantillonnage ultérieur, mais sa direction diffère légèrement de celle indiquée par le krigeage. L'intérêt d'une reconnaissance par étape, permettant de mieux délimiter les zones estimées avec une forte concentration, apparaît ici clairement.

Entre certaines taches mieux échantillonnées, sur le pourtour sud notamment, la variabilité locale des concentrations 
«krigées » est moindre, à cause du plus grand espacement entre les données, ayant comme conséquence une précision réduite.

Du fait de la prépondérance de la concentration maximale mesurée sur la valeur des combinaisons linéaires, un estimateur non linéaire comme le krigeage disjonctif peut ici compléter utilement le krigeage (Prechtel, 1998).

L'utilisation d'informations qualitatives faciles à acquérir et donc potentiellement abondantes, comme les relevés organoleptiques, ouvre une perspective intéressante pour améliorer la précision de l'estimation (Jeannée et de Fouquet, 2003).

\section{SÉLECTIVITÉ PRÉVUE ET RÉALISÉE}

\subsection{Sélection sur estimateur}

La délimitation de la zone à traiter a une grande influence sur le dimensionnement d'un chantier de dépollution. En cas d'excavation dans la zone ainsi définie, on procède au cours de l'avancement du chantier à un échantillonnage très fin, à maille plurimétrique, pour obtenir une estimation beaucoup plus précise des concentrations. Les sols sont alors sélectionnés et orientés d'après cette estimation «ultime», soit vers un traitement physicochimique ou biologique, ou une mise en décharge classée selon le niveau de pollution présumé, soit vers un stockage provisoire avant réemploi sur le site, pour des volumes supposés non pollués.

En cours de chantier, ou à l'issue de la dépollution, des écarts importants par rapport aux prévisions initiales ont déjà été constatés, les concentrations ou les volumes de sols pollués étant généralement sous-estimés. Ces écarts peuvent mettre en cause l'économie même du projet (Demougeot-Renard, 2002). Des contrôles ont parfois montré la présence de fortes concentrations non détectées lors du traitement du site.

Deux facteurs, présentés aux paragraphes suivants, contribuent à expliquer ces écarts inévitables, mais qui peuvent être réduits par des procédures d'échantillonnage, d'estimation puis de sélection adaptées (Matheron, 1984).

\section{L'effet d'information}

La délimitation initiale de la zone à dépolluer ainsi que la sélection puis l'orientation des volumes excavés ne s'effectuent jamais d'après les concentrations réelles, qui restent toujours inconnues, mais d'après les concentrations estimées. Lorsque l'estimation est imprécise, par exemple en présence d'une forte variabilité spatiale aux petites distances, la zone à traiter prévue lors du dimensionnement du chantier peut différer fortement de la zone réellement polluée, et les concentrations réelles peuvent s'écarter fortement des prévisions sur estimateur. Dans ce cas, lors du traitement du site, les erreurs de sélection des sols à dépolluer seront nombreuses.

\section{L'effet de support}

Pour des raisons techniques, la sélection porte sur des volumes (des «blocs ») de quelques mètres cubes à quelques dizaines de mètres cubes (par exemple, des blocs de $0,5 \mathrm{~m}$ d'épaisseur et $3 \times 3 \mathrm{~m}$ de côté). Or la concentration moyenne d'un bloc est la moyenne des concentrations de l'ensemble des échantillons fictifs (de quelques litres usuellement) contenus dans le bloc. La concentration moyenne d'un bloc est donc comprise entre la concentration maximale et la concentration minimale de tous les «échantillons » intérieurs au bloc. Dans un secteur, l'ensemble des blocs et l'ensemble des «échantillons» constituant ces blocs ont la même moyenne (égale à la concentration moyenne dans le secteur). Cependant, la concentration maximale des blocs est inférieure à celle des échantillons, et réciproquement, la concentration minimale des blocs est nécessairement supérieure à celle des échantillons.

Dans un chantier, la proportion des blocs présentant une concentration faible (respectivement forte) diffère donc nécessairement de la proportion donnée par l'histogramme expérimental des échantillons disponibles : les blocs présentent une plus forte proportion de concentrations intermédiaires, se rapprochant de la moyenne locale (fig. 10).

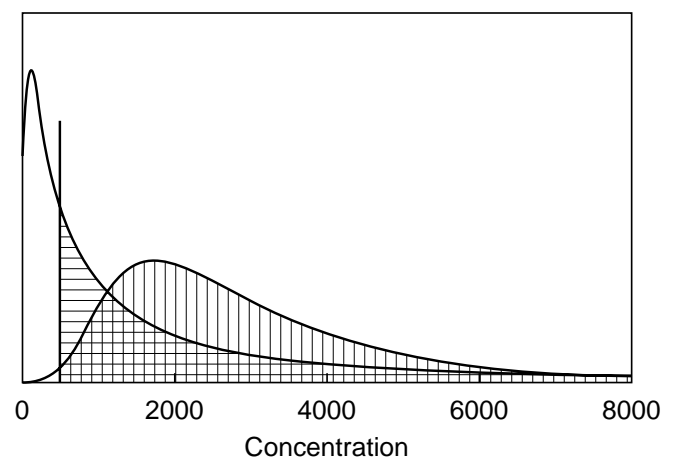

Figure 10

Histogramme des concentrations d'échantillons et de blocs. Les échantillons de concentration supérieure au seuil, ici égal à 500, correspondent aux zébrures horizontales (prévision), et les blocs aux zébrures verticales (dépollution optimale). La moyenne est égale à 3000 .

Histogram of the concentration of the samples and of the blocks. The samples with concentration greater than the threshold, here equal to 500, correspond to the horizontal hatching (prediction) and the vertical hatching (optimal remediation). The mean is equal to 3000.

Pour la caractérisation du site, les concentrations sont mesurées sur des échantillons dont le volume est fixé par des considérations étrangères aux objectifs de la dépollution (dimensions des outils de prélèvement, préparation et conditionnement des échantillons, etc.) Déduire la proportion des blocs, dont la concentration est supérieure à un seuil de 
qualité à partir de l'histogramme des échantillons, fournit une évaluation grossièrement erronée de la proportion des blocs dépassant effectivement le seuil. Selon le seuil considéré, l'histogramme des échantillons provoque une surestimation ou (plus fréquemment) une sous-estimation des tonnages de sols pollués.

Les techniques de «changement de support», initialement développées pour l'exploitation minière afin d'expliquer les écarts systématiques et défavorables constatés entre prévisions et résultats effectifs, permettent le calcul de l'histogramme des blocs. On en déduit le tonnage et la concentration moyenne des blocs dépassant un seuil donné (Rivoirard, 1995 ; Chilès et Delfiner, 1999), globalement sur le site, ou localement sur un secteur.

Sur un chantier de dépollution, effet de support et effet d'information se cumulant, les résultats sont toujours inférieurs aux prévisions.

\subsection{Remarques sur les seuils de qualité}

En pollution atmosphérique, la réglementation précise systématiquement le support temporel de référence pour les seuils de qualité, moyenne horaire ou moyenne annuelle, par exemple. Les seuils relatifs aux moyennes annuelles sont évidemment très inférieurs à ceux relatifs aux moyennes horaires, ces derniers concernant les épisodes de pollution aiguë.

En pollution de sol, la définition d'un seuil de qualité devrait toujours préciser le support spatial concerné, variable selon l'affectation future du site. En cas de fortes contraintes de qualité (pour l'implantation d'écoles ou de lotissements dont les jardins peuvent être utilisés comme potagers, par exemple), un support quasi ponctuel comme celui des échantillons semble pertinent, alors que des valeurs moyennes sur des «blocs» de plusieurs mètres cubes se justifieraient pour des activités moins sensibles (parkings, installations industrielles).

\subsection{Approximations pour la comparaison des prévisions à la « réalité »}

Nous plaçant dans le scénario d'un usage sensible du site, nous retenons désormais un support de sélection «ponctuel», correspondant au volume d'un échantillon. Une sélection «ponctuelle» correspond par exemple au ramassage manuel de sols pollués.

Nous examinons les écarts entre prévisions et résultats effectifs suivant deux critères :

- la régression des valeurs réelles sur les estimations, et ses conséquences sur l'évaluation des fortes concentrations ;

- la comparaison des prévisions aux résultats effectifs, en termes de tonnage et de concentration; ceci correspond exactement au calcul des «réserves récupérables » en exploitation minière.
Pour conserver aux résultats un caractère réaliste, nous comparons «prévisions » et «réalité » sur la zone des UP, dans laquelle les échantillons sont distants de 30 à $40 \mathrm{~m}$. Schématiquement, ces conditions correspondent au dimensionnement d'un chantier de dépollution, après resserrement de la maille de reconnaissance initiale, et avant l'échantillonnage dense effectué durant le traitement du site.

Pour examiner l'influence du modèle de variabilité spatiale, nous retenons successivement comme «estimateurs » les différents résultats de validation croisée. Les prévisions sont calculées à partir des estimations, et la réalité d'après la concentration mesurée des points ainsi sélectionnés. Dans cette comparaison, l'erreur de mesure sur les données est négligée.

Considérer les erreurs de validation croisée comme représentatives des erreurs d'estimation en des points non mesurés peut sembler pessimiste : la maille étant approximativement régulière dans la zone des UP, supprimer une donnée pour en faire l'estimation revient à augmenter en ce point la distance aux points expérimentaux les plus proches. L'ordre de grandeur des écarts observés par rapport aux prévisions reste cependant réaliste.

L'amplitude des erreurs de sélection commises lors de la dépollution peut être évaluée à partir de simulations géostatistiques conjointes des concentrations de «points» et de blocs, sur une maille resserrée (Demougeot-Renard, 2002). Cette technique permet de reproduire les erreurs d'échantillonnage, pour en évaluer les conséquences. Elle s'avère utile pour comparer quantitativement les résultats effectifs pour différentes mailles de reconnaissance.

\subsection{Régression entre concentration estimée et concentration mesurée}

Sur le nuage de corrélation des concentrations estimées et des concentrations mesurées, est reportée la «régression», qui est la moyenne de la concentration mesurée, par classe de valeur de la concentration estimée (fig. 11 et tableau 6). En l'absence de biais conditionnel, cette régression coïncide avec la première bissectrice.

Aux faibles concentrations, les moyennes par classe prévues sur estimateurs diffèrent peu des moyennes mesurées. Pour le modèle «mixte » et le modèle homogène, l'erreur relative sur la moyenne par classe reste cependant importante, et la dispersion des concentrations réelles par classe est considérable : à des valeurs voisines par la concentration estimée correspondent des concentrations réelles très différentes.

Pour la partition par mode, la régression expérimentale reste proche de la première bissectrice pour les concentrations (estimées) inférieures à 3000. Au-delà, la concentration «réelle » mesurée devient inférieure en moyenne aux prévisions. En sélectionnant les zones à dépolluer d'après la carte des concentrations estimées, on retire moins de pollution que prévu. 

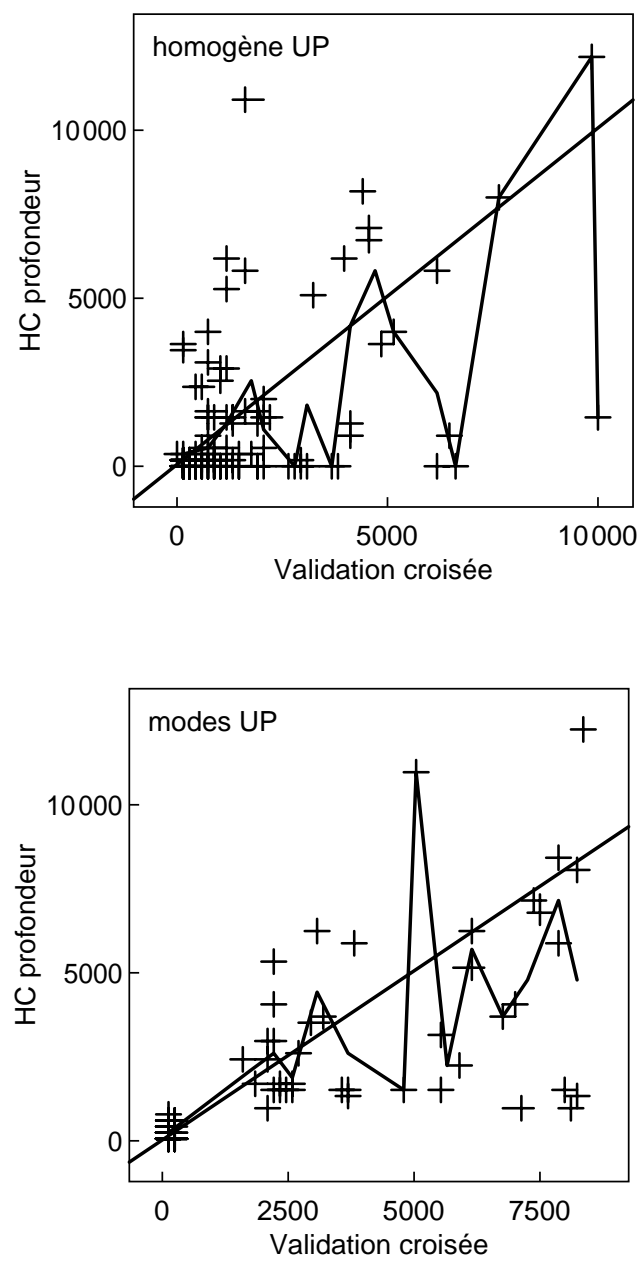

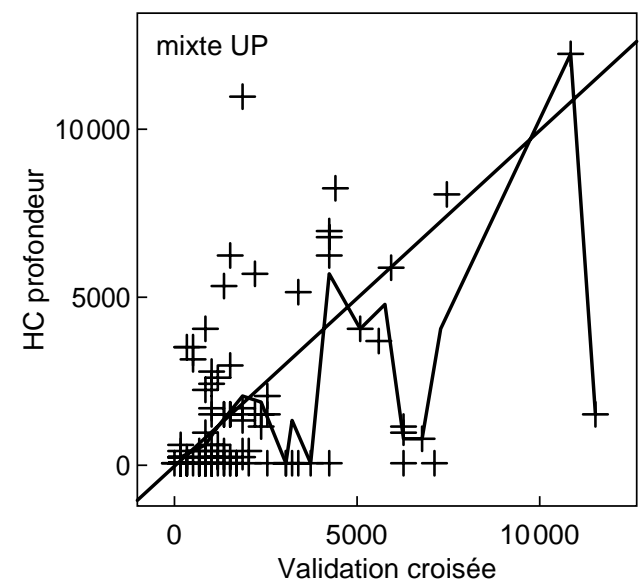

Figure 11

Nuage de corrélation des estimations par validation croisée (abscisse) et des concentrations mesurées (ordonnées), sur la zone des UP. Sont reportées la première bissectrice, ainsi que la régression de la concentration réelle sur la concentration estimée (courbe expérimentale). La régression expérimentale est calculée par classes de largeur 500. Pour la lisibilité des graphiques, les deux échantillons de plus fortes concentrations, largement sousestimés par la validation croisée, sont supprimés.

Correlation plot of the estimations by cross validation (abscissa) and measured concentrations (ordinate) in the former UP zone. Shown is the regression of the measured concentration on the estimated one (experimental curve), as welle as the first bisector. The experimental regression is calculated on classes of a width of 500. For better legibility of the graphics, the two samples with highest concentrations, which are clearly under estimated by cross validation, are suppressed.
Dans la pratique, lorsque l'estimation est imprécise, la concentration réelle inconnue peut différer fortement de son estimation, ces écarts provoquant de nombreuses erreurs de sélection. Une forte variabilité spatiale des concentrations à petite distance peut ainsi rendre impossible, à coût d'échantillonnage réaliste, une estimation précise pour le support de sélection retenu, par exemple des blocs de quelques mètres cubes. Dès le début de la reconnaissance du site, le resserrement local de la maille de reconnaissance est essentiel pour vérifier la faisabilité effective d'une dépollution fondée sur une sélection des sols selon leur concentration. Lorsque l'effet d'information rend la sélection inefficace, un traitement non sélectif peut se révéler plus fiable et également meilleur marché, car il économise alors le coût de la reconnaissance.

\subsection{Prévisions de " récupérable »}

Prévoir le volume de sols à dépolluer (dont la concentration réelle ou estimée dépasse un seuil fixé) et la quantité de polluant présent dans ces volumes est un problème analogue à celui rencontré en exploitation minière. Reprenant la terminologie minière, nous désignons par:

- bloc, l'unité minimale de sélection lors de la dépollution, par exemple des volumes de $2 \times 2 \times 0,5 \mathrm{~m}$;

- coupure, le seuil de concentration au-delà duquel un «bloc » est sélectionné comme pollué ;

- minerai, le volume (ou à un facteur près, le tonnage) des blocs ainsi sélectionnés;

- métal, la quantité de polluant présent dans le minerai sélectionné. À un facteur près, le métal est le produit du minerai par la concentration moyenne des blocs sélectionnés. Dans le cas d'une pollution métallique, le «métal» correspond effectivement, à un facteur près, à la masse de polluant présente dans le minerai.

En pratique, la sélection s'effectue toujours d'après les concentrations estimées. Nous comparons donc les prévisions, calculées sur les concentrations estimées, au « réalisé », qui correspond aux valeurs réelles des blocs ainsi sélectionnés. 


\section{TABLEAU 6}

Régression entre concentrations estimées par validation croisée et concentration mesurée, pour la zone des UP. A : modes à 800 ; $\mathrm{B}$ : populations mixtes ; $\mathrm{C}$ : population « homogène »

Regression between predicted by cross validation, and measured concentrations, for the former UP zone. A: modes at 800; B: mixed populations; C: "homogeneous" population

\begin{tabular}{l|c|c|c|c|c}
\hline \multirow{2}{*}{ Classe de valeur } & \multicolumn{2}{|c|}{ Estimation } & \multicolumn{2}{c}{$\begin{array}{c}\text { Concentration } \\
\text { mesurée }\end{array}$} \\
\hline \multirow{2}{*}{0} & & Moyenne & $\sigma$ & Moyenne & $\sigma$ \\
\hline $0-500$ & A & 147 & 41 & 149 & 151 \\
& B & 175 & 102 & 306 & 744 \\
& C & 281 & 104 & 363 & 815 \\
\hline $500-1000$ & A & - & - & - & - \\
& B & 808 & 112 & 571 & 1050 \\
& C & 773 & 131 & 658 & 1012 \\
\hline $1000-2000$ & A & 1738 & 93 & 2057 & 353 \\
& B & 1381 & 287 & 1447 & 2188 \\
& C & 1380 & 304 & 1626 & 2429 \\
\hline $2000-3000$ & A & 2317 & 189 & 2323 & 1174 \\
& B & 2464 & 274 & 5026 & 9459 \\
& C & 2397 & 329 & 3859 & 9211 \\
\hline $3000-4000$ & A & 3438 & 302 & 3301 & 1906 \\
& B & 3320 & 191 & 1127 & 2005 \\
& C & 3397 & 314 & 1123 & 2007 \\
\hline
\end{tabular}

En italique, les classes comportant les deux concentrations maximales mesurées dans cette zone, retirées de la figure 11. Pour la partition par mode, ces valeurs étant estimées supérieures à 4000 n'apparaissent pas dans le tableau. Pour cette partition, aucune concentration n'est estimée entre 500 et 1000.

In italics: data including the two largest concentrations values, omitted in Figure 11. For the partition by mode, the values estimated greater than 4000 do not show up in the table. In that case, no concentration has been estimated in the range of 500 to 1000.

Nous supposons la sélection «libre», les blocs étant sélectionnés d'après leur seule concentration estimée, sans tenir compte des contraintes d'accessibilité des zones à traiter. L'évaluation de l'incidence des contraintes techniques, comme l'accès ou la stabilité des parements, sur les résultats de sélection a été abordée en contexte minier par simulations d'exploitation sur modèles numériques (simulations géostatistiques), dès la fin des années 1970 (Deraisme et al., 1984).

Les erreurs d'estimation provoquent des erreurs de sélection : la concentration moyenne effective et le métal associé diffèrent des prévisions, dans la zone supposée polluée comme dans la zone supposée non polluée. Les erreurs de sélection sont de deux types : estimateur supérieur à la coupure et concentration réelle inférieure, ou réciproquement.

Ces erreurs sont d'autant plus importantes que :

- la dispersion des concentrations réelles par classe de valeur estimée est importante, ce qui se traduit par une forte dispersion verticale du nuage de corrélation des valeurs mesurées sur les valeurs estimées ;
- la régression des concentrations mesurées sur les concentrations estimées s'écarte de la première bissectrice.

Sur les anciennes UP, les statistiques des erreurs de validation croisée étant proches pour le modèle mixte et le modèle homogène, nous conservons comme « référence »le modèle mixte. Nous nous plaçons ainsi dans le cas où les zones de fortes concentrations sont délimitées assez approximativement, par exemple en raison d'une connaissance lacunaire de l'historique du site.

La figure 12a présente, pour les trois modèles, le pourcentage de métal effectivement récupéré en fonction du pourcentage de minerai sélectionné d'après les concentrations estimées. Est également reportée la courbe de la sélection «idéale » effectuée sans erreur sur les concentrations mesurées, courbe nécessairement concave (Matheron, 1984).

Pour la sélection ponctuelle envisagée ici, chaque échantillon compte comme une unité. Le minerai total (correspondant à la coupure 0) présent dans la zone des UP est égal à 128 .

\section{TABLEAU 7}

Sélection « idéale » sur concentration réelle, et comparaison au résultat prévu $(\mathrm{P})$ et réalisé $(\mathrm{R})$ par le modèle mixte. UP

"Ideal" selection, based on the real concentration, and comparison to the predicted value $(P)$ and the realized value $(R)$ after selection using the mixed model. Former UP zone

\begin{tabular}{|c|c|c|c|}
\hline UP & & Idéal & Mixte \\
\hline & \multicolumn{3}{|c|}{ Coupure 500} \\
\hline Points sélectionnés & & 47 & 88 \\
\hline \multirow[t]{2}{*}{ Métal récupéré } & $\mathrm{P}$ & & $21110^{3}$ \\
\hline & $\mathrm{R}$ & $20510^{3}$ & $20310^{3}$ \\
\hline \multirow[t]{3}{*}{ Concentration moyenne } & $\mathrm{P}$ & & 2396 \\
\hline & $\mathrm{R}$ & 4358 & 2306 \\
\hline & \multicolumn{3}{|c|}{ Coupure 1000} \\
\hline Points sélectionnés & & 40 & 65 \\
\hline \multirow[t]{2}{*}{ Métal récupéré } & $\mathrm{P}$ & & $19210^{3}$ \\
\hline & $\mathrm{R}$ & $20010^{3}$ & $19010^{3}$ \\
\hline \multirow[t]{3}{*}{ Concentration moyenne } & $\mathrm{P}$ & & 2957 \\
\hline & $\mathrm{R}$ & 4993 & 2919 \\
\hline & \multicolumn{3}{|c|}{ Coupure 2000} \\
\hline Points sélectionnés & & 26 & 31 \\
\hline \multirow[t]{2}{*}{ Métal récupéré } & $\mathrm{P}$ & & $14510^{3}$ \\
\hline & $\mathrm{R}$ & $17910^{3}$ & $14110^{3}$ \\
\hline \multirow[t]{2}{*}{ Concentration moyenne } & $\mathrm{P}$ & & 4685 \\
\hline & $\mathrm{R}$ & 6879 & 4535 \\
\hline
\end{tabular}

$\mathrm{P}$ : prévision sur estimateur, $\mathrm{R}$ : récupéré (résultat réel après sélection sur estimateur).

$P$ : prediction on the estimated values, $R$ : "recovered" i.e. real result after selection on the estimated concentrations. "Coupure" denotes the cutoff. 


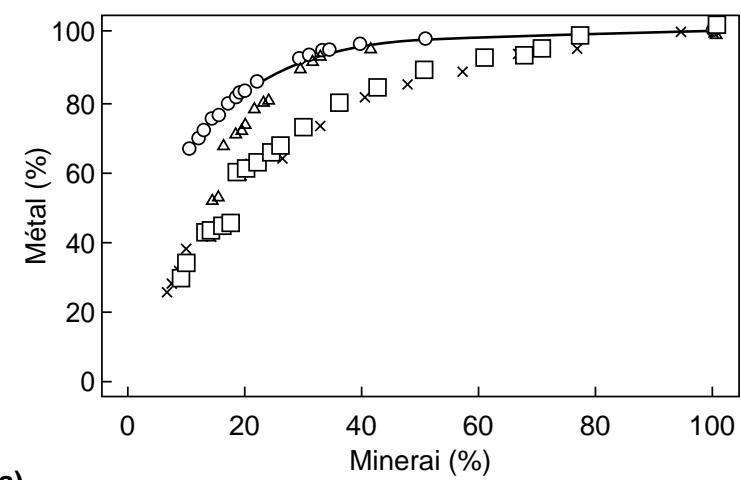

a)

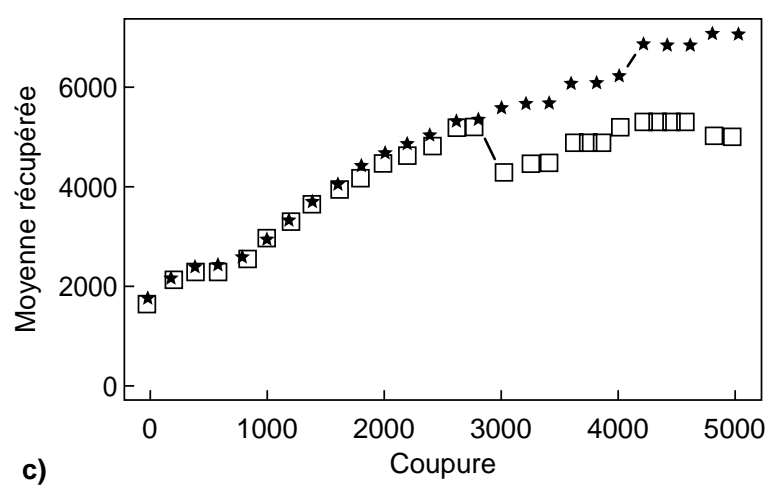

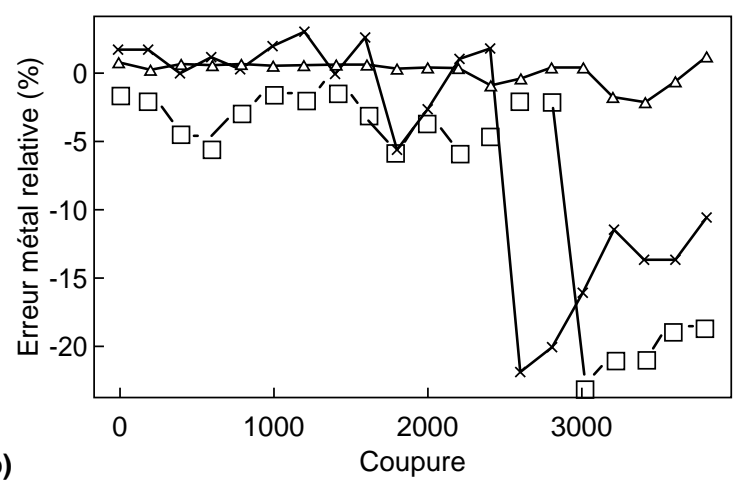

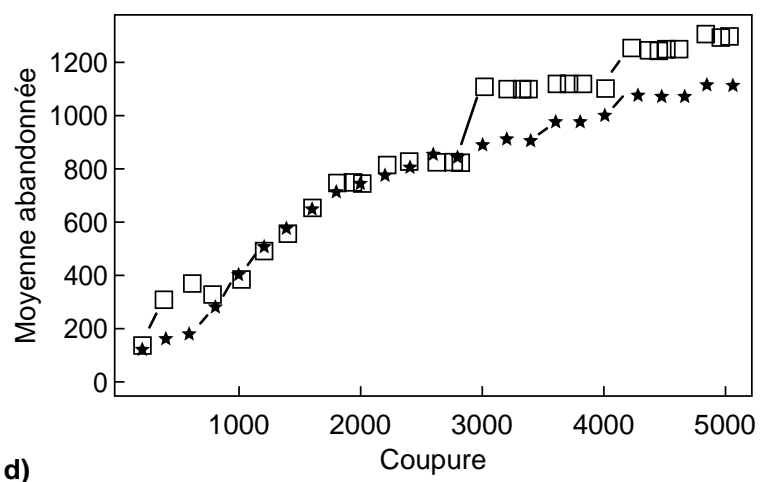

Figure 12

Calcul de récupérable sur la zone des UP. a) «métal » effectivement récupéré en fonction du tonnage de minerai sélectionné. Cercles : sélection idéale sur les concentrations mesurées ; triangles : partition par mode ; carrés : modèle mixte ; croix : modèle homogène. b) erreur relative sur le métal en fonction de la coupure. L'erreur relative est calculée comme la différence entre le métal effectivement récupéré et le métal prévu sur estimateur, divisée par le métal prévu (mêmes symboles qu'en a). c) concentration moyenne prévue sur estimateur (étoiles) et réalisée (carrés) dans la zone supposée polluée. Estimation en modèle mixte. d) concentration moyenne prévue sur estimateur (étoiles) et réalisée (carrés) dans la zone supposée non polluée. Estimation en modèle mixte.

Calculation of the "recoverable » amounts on the former UP. a) effectively recovered "metal" as a function of the tonnage of selected mineral resources. Circles: ideal selection, based on the measured concentrations; triangles: partition by mode; squares: mixed model; crosses: homogeneous model. b) relative error for the metal as a function of the cutoff. The relative error is calculated as the difference of the effectively recovered "metal" and the predicted "metal" contents calculated on the estimation, divided by the predicted "metal" content. The symbols are the same as in a). c) mean predicted concentration calculated on the estimation (stars) and effectively recovered value (squares) in the zone supposed to be polluted. Estimation on the basis of the mixed model. d) mean predicted concentration calculated on the estimation (stars) and effectively recovered value (squares) in the zone supposed to be not polluted. Estimation on the basis of the mixed model.

À même tonnage de minerai, le métal récupéré est maximum pour la sélection idéale, qui cible les plus fortes concentrations. Le modèle mixte et le modèle homogène fournissent des résultats voisins, avec une récupération inférieure à celle obtenue à partir des estimations de la partition par mode.

Les écarts les plus importants à la sélection idéale s'observent aux fortes coupures, donc aux faibles tonnages de minerai, les fortes concentrations étant mal réestimées, et par suite, mal localisées. À minerai fixé, l'écart à la sélection «idéale» est important, avec une perte dépassant $20 \%$ du métal total lorsque le minerai est inférieur à $40 \%$ du total en place (tableau 7).
La figure $12 \mathrm{~b}$ montre, pour les trois estimateurs, l'erreur relative sur le métal prévu, calculée comme l'écart entre prévision et réalisation, rapporté à la prévision; la variable en abscisse est la coupure. Aux coupures élevées, le métal prévu est faible et les erreurs relatives augmentent, d'autant plus qu'elles portent sur les fortes concentrations, notamment pour le modèle mixte. Pour la partition par mode, ainsi que pour le modèle homogène, les erreurs relatives sont faibles. Mais ces faibles écarts entre métal (ou concentration moyenne) prévu et effectif ne signifient pas que la sélection est proche de l'optimum. En effet, la moyenne des concentrations sélectionnées peut s'approcher de la moyenne 
prévue sur estimateur dans le cas où des concentrations faibles sélectionnées par erreur compensent des concentrations sélectionnées avec raison, mais dont la valeur est supérieure aux prévisions. Comme on le voit pour le modèle homogène, les faibles écarts entre métal prévu et réalisé sont ainsicompatibles avec une récupération incomplète du métal à tonnage minerai fixé.

L'écart entre concentration moyenne prévue et concentration réalisée est présenté pour l'estimation en modèle mixte, dans la zone sélectionnée (fig. $12 c$ ) et dans le complémentaire supposé non pollué (figure 12d). Malgré les imperfections de cet estimateur, on observe une bonne concordance entre concentration (ou métal) prévue et réalisée pour une large gamme de coupures. Cependant, l'effet d'information est évidemment présent: abandon de concentrations réelles supérieures au seuil, ou sélection inutile de concentrations réelles inférieures au seuil.

Le tableau 7 confirme ces observations. Aux coupures 500 et 1000 , le métal récupéré est proche du résultat de la sélection idéale, mais aux trois coupures considérées, le nombre de points sélectionnés (le minerai) est largement supérieur à celui de la sélection «idéale »: des blocs de concentration inférieure à la coupure sont inutilement sélectionnés comme pollués. La concentration moyenne dans les volumes sélectionnés, proche des prévisions, est largement inférieure à celle de la section idéale.

Remarque: sur les estimations, la concentration moyenne prévue des points passant la coupure est nécessairement une fonction croissante de la coupure; ce n'est plus nécessairement le cas pour la moyenne réelle (fig. 12).

En présence d'une forte variabilité spatiale à petite distance, la mauvaise précision des estimations peut rendre toute sélectivité illusoire. Dans le cas limite d'un effet de pépite pur, la moyenne est identique dans la zone sélectionnée sur estimateur, et dans son complémentaire. Dans ce cas particulier, aucune sélection n'est plus possible, et le resserrement de la maille de reconnaissance lors de la dépollution devient donc inutile.

En pratique, ne pas détecter des blocs de concentration supérieure à la coupure peut générer un risque sanitaire, mais aussi financier, en cas de contrôle en fin d'opérations. Sélectionner un tonnage largement supérieur au tonnage «idéal » provoque des surcoûts, dus au traitement de blocs non pollués. Un écart important entre la concentration moyenne prévue pour les blocs sélectionnés et leur moyenne réelle peut rendre le procédé de dépollution inadapté.

\section{5 ÉLÉMENTS DE MODÉLISATION}

Jusqu'à présent, les erreurs de sélection ont été quantifiées empiriquement, sans autre modélisation que le variogramme de la concentration, nécessaire au krigeage. Nous rappelons brièvement quelques éléments de modélisation pour le calcul des incertitudes, précédemment appliqués à des pollutions organiques (Jeannée, 2001) ou métalliques (Demougeot-Renard, 2002), et nous donnons des indications permettant d'effectuer des prévisions de façon simple mais rigoureuse.

Pour l'estimation des concentrations (le krigeage), un modèle de variogramme est suffisant (avec les hypothèses sur l'homogénéité ou l'hétérogénéité du site, ainsi que le voisinage de krigeage). Les calculs non linéaires comme l'évaluation d'une probabilité de dépassement de seuil nécessitent une modélisation plus poussée, soit de la loi bivariable (la loi de probabilité conjointe des concentrations en deux points quelconques), soit de la loi multivariable (la loi de probabilité conjointe des concentrations en tout ensemble fini de points). Un modèle souvent bien adapté (mais pas toujours) est le modèle « multigaussien anamorphosé ». La concentration $Z(x)$ est considérée comme la transformée par une fonction croissante (ou anamorphose) $\varphi$ d'une fonction aléatoire de loi spatiale gaussienne réduite $Y(x)$. En pratique, plusieurs tests permettent de vérifier la compatibilité de cette hypothèse aux données expérimentales (Matheron, 1984 ; Rivoirard, 1995 ; Chilès et Delfiner, 1999).

Le modèle gaussien anamorphosé permet de construire des simulations conditionnelles de la concentration, qui sont des modèles numériques reproduisant la loi spatiale des concentrations et passant par les valeurs expérimentales aux points de données, en tenant éventuellement compte d'une erreur de mesure. Les simulations conjointes des concentrations de points et de blocs permettent ensuite d'évaluer le métal récupéré pour différentes stratégies d'échantillonnage (Demougot-Renard et al., 2002).

Dans la littérature, la probabilité de dépassement de seuil est souvent calculée en construisant un grand nombre de simulations conditionnelles de la concentration (ponctuelle ou moyenne par bloc selon le cas), puis en comptant point par point la proportion des simulations dépassant le seuil. $\mathrm{Ce}$ procédé, correct du point de vue théorique mais lourd en temps de calcul, est avantageusement remplacé par un calcul direct, reposant exactement sur le même modèle probabiliste. En effet, désignons par $Z_{\alpha}$ les données expérimentales, et par $Y_{\alpha}$ leurs transformées gaussiennes. Pour simplifier le support retenu pour l'estimation est celui des échantillons (l'estimation est «ponctuelle»). Pour un seuil $s$, la probabilité que la concentration au point $x$ dépasse le seuil, conditionnellement aux mesures, s'écrit :

$$
\begin{aligned}
P(Z(x) & \left.>s \mid Z_{\alpha}, \alpha=1, \ldots, n\right) \\
& =P\left(\varphi(Y(x))>s \mid Y_{\alpha}, \alpha=1, \ldots, n\right) \\
& =P\left(Y(x)>\varphi^{-1}(s) \mid Y_{\alpha}, \alpha=1, \ldots, n\right)
\end{aligned}
$$

$Y$ étant une fonction aléatoire de loi spatiale gaussienne, la loi conditionnelle de $Y(x)$ est connue. Elle s'écrit simplement comme la somme de $Y^{*}(x)$, krigeage à moyenne nulle de $Y(x)$ 
par les $Y_{\alpha}$, et d'un «résidu » $\sigma_{K}(x) R(x), \sigma_{K}(x)$ désignant l'écart type de krigeage associé, et $R(x)$ une fonction aléatoire d'histogramme gaussien, d'espérance nulle et de variance unité. On a ainsi :

$$
\begin{aligned}
P(Z(x) & \left.>s \mid Z_{\alpha}, \alpha=1, \ldots, n\right) \\
& =P\left(Y^{*}(x)+\sigma_{K}(x) R(x)>\varphi^{-1}(s)\right)
\end{aligned}
$$

Le « résidu » $R(x)$ étant indépendant de $Y^{*}(x)$, on en déduit :

$$
\begin{aligned}
P(Z(x) & \left.>s \mid Z_{\alpha}, \alpha=1, \ldots, n\right) \\
& =1-G\left(\frac{\varphi^{-1}(s)-Y^{*}(x)}{\sigma_{K}}\right)
\end{aligned}
$$

$G$ désignant la fonction de répartition d'une variable gaussienne réduite, qui est connue.

En résumé, la probabilité de dépassement de seuil est donnée par le krigeage et l'écart type de krigeage des transformées gaussiennes.

Les quantiles locaux s'obtiennent de façon analogue: soit $s_{p}(x)$ le quantile d'ordre $p$ de $Z(x)$, c'est-à-dire la valeur dépassée avec une probabilité de $1-p$ (par exemple, $S_{0,90}(x)$ est la concentration dépassée localement au point $x$ dans $1-p$ $=10 \%$ des cas) ; notant $\tau_{p}(x)$ le quantile d'ordre de la loi conditionnelle des transformées gaussiennes, celui-ci est déterminé par :

$$
\begin{aligned}
1-p & =P\left(Z(x)>S_{p}(x) \mid Z_{\alpha}, \alpha=1, \ldots, n\right) \\
& =P\left(Y^{*}(x)+\sigma_{K}(x) R(x)>\tau_{p}(x)\right) \\
& =1-G\left(\frac{\tau_{p}(x)-Y^{*}(x)}{\sigma_{K}(x)}\right)
\end{aligned}
$$

d'où $\tau_{p}(x)=\sigma_{K}(x) G^{-1}(p)+Y^{*}(x)$, ce qui détermine le quantile local $s_{p}(x)$ de la concentration par la fonction d'anamorphose : $s_{p}=\varphi\left(\tau_{p}\right)$.

Effectuer un grand nombre de simulations, pour déterminer empiriquement point par point la probabilité de dépassement d'un seuil donné (par le rapport du nombre de «cas favorables » au nombre de simulations), ou bien la valeur dépassée avec une probabilité de $1-p$, consiste à utiliser exactement les mêmes hypothèses que les calculs précédents. Le calcul sur simulations revient à approcher numériquement ces grandeurs directement fournies par le modèle probabiliste.

Pour des calculs plus complexes, faisant intervenir notamment des contraintes de nature géométrique pour la sélection des blocs, ou plusieurs supports simultanément, le recours aux simulations reste généralement inévitable. C'est par exemple le cas lorsque l'on veut comparer quantitativement différents scénarios de dépollution (dimension des unités de sélection, échantillonnage pendant le chantier de dépollution).

\section{CONCLUSION}

La faisabilité et l'utilité des études géostatistiques pour la quantification des pollutions sont désormais démontrées, aussi bien pour des pollutions organiques que métalliques. Préliminairement à toute modélisation, l'analyse exploratoire des données est une étape indispensable qui permet de baser la modélisation sur l'information expérimentale, en particulier la localisation des niveaux de concentrations, et leur variabilité spatiale. Par exemple, l'hétérogénéité des concentrations sur un site est détectable par la multimodalité de l'histogramme des concentrations ou de leur logarithme.

La modélisation de la structure spatiale des concentrations a une réelle influence sur l'efficacité de la sélection, notamment pour le dimensionnement d'un chantier, ou pour la conduite des opérations de dépollution.

Comme nous l'avons montré expérimentalement, la cohérence de la moyenne prévue et de la moyenne effective des concentrations sélectionnées ne garantit pas la qualité de la sélection. Le tonnage de polluant sélectionné peut en effet être largement inférieur au tonnage réellement en place, ou le tonnage de minerai sélectionné peut être inutilement élevé.

Le calcul de la probabilité de dépassement de seuil, qui quantifie les incertitudes par rapport à la concentration de coupure, constitue un complément utile aux cartes de concentrations estimées. Ce calcul, comme celui des quantiles de la concentration, peut s'effectuer directement et de façon rigoureuse, sans recourir aux techniques de simulation.

\section{REMERCIEMENTS}

Nous remercions MM. Roger Camps et Alain Perez (TоTAL) pour leurs avis durant ce travail.

\section{RÉFÉRENCES}

Beucher, H., Doligez, B. et Yarus, J.M. (à paraître) Modeling Complex Reservoirs with Multiple Conditional Techniques: a Practical Approach to Reservoir Characterization. AAPG, Stochastic Modelling II.

Chilès, J.P. et Delfiner, P. (1999) Geostatistics: Modeling Spatial Uncertainty. In: N.Y. Wiley ed., Wiley Series in Probability and Statistics: Applies Probability and Statistics Section.

Demougeot-Renard, H. (2002) De la reconnaissance à la réhabilitation des sols industriels pollués : estimations géostatistiques pour une optimisation multicritère. Thèse de doctrat $\mathrm{n}^{\circ} 14615$, ETHZ.

Demougeot-Renard, H., de Fouquet, C. et Fritsch, M. (2002) Optimizing Sampling for Acceptable Accuracy Levels on Remediation Volume and Cost Estimations. In: Proceedings of the 4th European Conference on Geostatistics for Environmental Applications, held in Barcelona, 27-29 December.

Deraisme, J., de Fouquet, C., Fraisse, H. (1984) Geostatistical Orebody Model for Computer Optimization of Profits from Different Underground Mining Methods. In: Proc. 18th APCOM Symp., London. Institution of Mining and Metallurgy, 583-590. 
Jeannée, N. (2001) Caractérisation géostatistique de pollutions industrielles de sols. Cas des HAP sur d'anciens sites de cokeries. Thèse de doctorat en géostatistique, École des mines de Paris.

Jeannée, N. et de Fouquet, C. (2003) Apport d'informations qualitatives pour l'estimation des teneurs en milieux hétérogènes: cas d'une pollution de sols par des hydrocarbures aromatiques polycycliques (HAP). Comptes rendus Géoscience, 335, 5, 441449.

Leenaers, H., Okx, J.P. et Burrough, P.A. (1989) Co-kriging: an accurate and inexpensive means of mapping floodplain soil pollution by using elevation data. In: M. Arsmtrong ed., Geostatistics, Dordrecht: Kluwer, 1, 371-382.

Matheron, G. (1970) La théorie des variables régionalisées, et ses applications. Cahiers du CMM, fascicule 5, École des mines de Paris.

Matheron, G. (1984) The Selectivity of the Distributions and the "Second Principle of Geostatistics". Proceedings, 2nd NATO ASI "Geostatistics for Natural Resources Characterization", Tahoe, Verly, G. et al., Eds, (1984). D. Reidel Publ. Co., Dordrecht, Holland.
Matheron, G. (1989) The Internal Consistency of Models in Geostatistics. In: M. Arsmtrong ed., Geostatistics, Dordrecht: Kluwer, 1, 21-38.

Papritz, A. et Dubois, J.P. (1999) Mapping Heavy Metals in Soils by (Non-)Linear Kriging: an Empirical Validation. In: GómezHernández, J., Soares, A., Froidevaux, R., eds., geoENV II Geostatistics for Environmental Application, Kluwer Academic Publishers.

Prechtel, A. (1998) La pollution d'un site par des hydrocarbures. Aspects de la modélisation hydrogéologique et étude géostatistique. Mémoire de DEA, Centre de géostatistique, Ecole des mines de Paris.

Rivoirard, J. (1994) Introduction to Disjunctive Kriging and Non linear Geostatistics, Clarendon press, Oxford.

Scholz, M., Oliver, M.A., Webster, R., Loveland, P.J. et Mcgrath, S.P. (1999) Sampling to Monitor Soil in England and Wales. In: Gómez-Hernández, J., Soares, A., Froidevaux, R., eds., geoENV II - Geostatistics for Environmental Application. Kluwer Academic Publishers.

Manuscript définitif remis en juin 2004 